\title{
Ultra-luminous high-redshift quasars from SkyMapper - II. New quasars and the bright end of the luminosity function
}

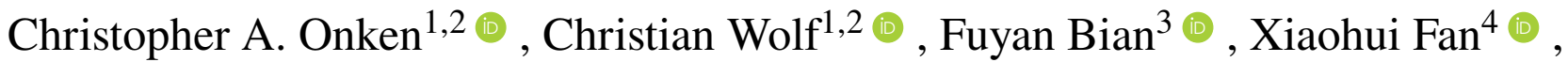 \\ Wei Jeat Hon ${ }^{5}$, David Raithel ${ }^{1}$, Patrick Tisserand ${ }^{6} \odot$, Samuel Lai ${ }^{1}$ \\ ${ }^{1}$ Research School of Astronomy and Astrophysics, Australian National University, Canberra ACT 2611, Australia \\ ${ }^{2}$ Centre for Gravitational Astrophysics, Australian National University, Canberra ACT 2600, Australia \\ ${ }^{3}$ European Southern Observatory, Alonso de Córdova 3107, Casilla 19001, Vitacura, Santiago 19, Chile \\ ${ }^{4}$ Steward Observatory, University of Arizona, 933 North Cherry Avenue, Tucson, AZ 85721, USA \\ ${ }^{5}$ School of Physics, University of Melbourne, Parkville, Victoria 3010, Australia \\ ${ }^{6}$ Sorbonne Universités, UPMC Univ Paris 6 et CNRS, Institut d'Astrophysique de Paris, 98 bis bd Arago, F-75014 Paris, France
}

Accepted XXX. Received YYY; in original form ZZZ

\begin{abstract}
We search for ultra-luminous Quasi-Stellar Objects (QSOs) at high redshift using photometry from the SkyMapper Southern Survey Data Release 3 (DR3), in combination with 2MASS, VHS DR6, VIKING DR5, AllWISE, and CatWISE2020, as well as parallaxes and proper motions from Gaia DR2 and eDR3. We report 142 newly discovered Southern QSOs at $3.8<z<5.5$, of which 126 have $M_{145}<-27$ ABmag and are found in a search area of $14486 \mathrm{deg}^{2}$. This Southern sample, utilising the Gaia astrometry to offset wider photometric colour criteria, achieves unprecedented completeness for an ultra-luminous QSO search at high redshift. In combination with already known QSOs, we construct a sample that is $>80$ per cent complete for $M_{145}<-27.33 \mathrm{ABmag}$ at $z=4.7$ and for $M_{145}<-27.73 \mathrm{ABmag}$ at $z=5.4$. We derive the bright end of the QSO luminosity function at restframe $145 \mathrm{~nm}$ for $z=4.7-5.4$ and measure its slope to be $\beta=-3.60 \pm 0.37$ and $\beta=-3.38 \pm 0.32$ for two different estimates of the faint-end QSO density adopted from the literature. We also present the first $z \sim 5$ QSO luminosity function at restframe $300 \mathrm{~nm}$.
\end{abstract}

Key words: galaxies: active - quasars: general - early Universe

\section{INTRODUCTION}

Supermassive black holes can be observed across vast distances, provided they accrete matter at a sufficient rate. Radiation released in the accretion process makes these objects the most luminous in the entire Universe. As long as they are not obscured by local dust, they will be seen as Quasi-Stellar Objects (QSOs) with characteristic spectral signatures. Ultra-luminous QSOs point us to the most massive and fastest growing black holes in the Universe. The demographics of this type of object are of particular interest at high redshift, an era in the early universe in which black holes undergo their most dramatic and least-explained growth.

As these most extreme objects are intrinsically rare, any search for them has always been for the proverbial needles in a haystack. Over the last two decades, useful samples of high-redshift ultraluminous QSOs have been detected, supported by a range of massive data sets including the iconic Sloan Digital Sky Survey (SDSS; York et al. 2000), and later supported by all-sky data from the Widefield Infrared Survey Explorer (WISE; Wright et al. 2010). Two more useful steps have been the recent addition of a Southern analogue to SDSS, the SkyMapper Southern Survey (SMSS; Wolf et al. 2018a; Onken et al. 2019) as well as infrared sky sur- veys such as the VISTA Hemisphere Survey (VHS; McMahon et al. 2013). At all steps in this journey, candidate lists for the rare high-redshift QSOs were swamped with the tails of the distribution from cool, red stars in our own Milky Way Galaxy. A major simplification of these searches was delivered by the Gaia satellite mission (Gaia Collaboration et al. 2018) of the European Space Agency (ESA); Gaia measured proper motions and parallaxes for a billion objects. This data set revealed the cool-star nature for a large fraction of the candidates, allowing effective cleaning of the candidate lists. Within two days of the release of Gaia DR2, Wolf et al. (2018b) identified the most luminous known QSO, SMSS J215728.21-360215.1 (a.k.a. SMSS J2157-3602) at $z=4.692$, which has since been shown to be powered by a supermassive black hole with 34 billion solar masses (Onken et al. 2020).

As ultra-luminous QSOs at high redshift are extremely rare, the size and completeness of a sample matters for any inference on the bright end of their luminosity function, their evolution with cosmic time in the early universe, their contribution to reionisation in the early universe, and the evolution of their host galaxies and early galaxies in general. These objects also lend a helping hand 
to studies of the intergalactic medium and the build-up of chemical elements traced with absorption lines in foreground galaxies (Ryan-Weber et al. 2009; Simcoe et al. 2011). For all these reasons, enlarging the existing samples is a worthwhile undertaking.

Currently, the best reference for the bright end of the QSO luminosity function (LF) at redshift $\sim 5$ is the work by Yang et al. (2016, hereafter Y16), which is based on a QSO sample from Wang et al. (2016, hereafter W16). Although various other works in the literature have updated the $z \sim 5$ QSO LF (e.g. McGreer et al. 2018; Kim et al. 2020; Niida et al. 2020), progress in this area is currently focused on the persistent uncertainties at the faint end. At the bright end, the exploitation of new data sources such as Pan-STARRS (Chambers et al. 2016) led to the discovery of many new QSOs (Yang et al. 2019; Schindler et al. 2019a), but no updates to the LF bright-end parameters. The work by Y16 inherited from its data source, the SDSS, a focus on the Northern hemisphere, and searching in the South offers an opportunity to at least double the sample and refine the sparsely populated bright end of the LF.

Hence, we had set out to discover ultra-luminous high-redshift QSOs in the Southern sky, armed with a combination of the SMSS, the AllWISE data set ${ }^{1}$ and Gaia DR2. The new Gaia data allowed Wolf et al. (2020, hereafter Paper I) to push QSO colour selection criteria closer to the main stellar locus, relative to the work by W16 and Y16, given that most stars could be identified and removed from the list by proper motions. The extended colour selections then revealed the rare QSOs with higher completeness than before. Similarly, Calderone et al. (2019) found that searches for QSO at all redshifts based on machine learning benefit from the Gaia data.

Paper I published a sample of 21 bright $z>4$ QSOs, selected from SMSS DR2 with $i_{\mathrm{PSF}}<18.2 \mathrm{ABmag}$. In this paper, we extend the search with SMSS DR3, reaching deeper in magnitude and lower in Galactic latitude, and purify the selection by adding JHK photometry from VHS and the VIKING Survey (Edge et al. 2013), filling in data from the shallower 2 Micron All-Sky Survey (2MASS; Skrutskie et al. 2006) where needed. We present the results of our spectroscopic follow-up of candidates down to $z_{\mathrm{PSF}} \approx 19 \mathrm{ABmag}$. We examine our evolving selection criteria in light of known highredshift QSOs in the Southern sky and suggest selection rules going forward. We compare the results of our search to previous campaigns in the Northern sky, and provide an update to the bright end of the high-redshift QSO luminosity function in the often quoted restframe $145 \mathrm{~nm}$ band. Finally, we present, for the first time, direct measurements for the bright end of the luminosity function at restframe $300 \mathrm{~nm}$.

Sect. 2 describes the data sources from which we construct our set of QSO candidates. In Sect. 3, we discuss properties of the known QSOs and the selection rules we adopt. In Sect. 4, we describe our spectroscopic follow-up of candidates, present the list of highredshift QSOs we found, and discuss the completeness of the current sample. In Sect. 5, we construct luminosity functions and examine their evolution. Throughout the paper, we use Vega magnitudes for Gaia and IR data, and AB magnitudes for the SkyMapper passbands: griz. We adopt a flat $\Lambda$ CDM cosmology with $\Omega_{\mathrm{m}}=0.3$ and a Hubble-Lemaître constant of $H_{0}=70 \mathrm{~km} \mathrm{sec}^{-1} \mathrm{Mpc}^{-1}$.

\footnotetext{
${ }^{1}$ Explanatory Supplement to AllWISE Data Release, http://wise2.ipac.caltech.edu/docs/release/allwise/expsup
}

\section{SEARCH AREA AND DATA SOURCES}

We start from the master catalogue ${ }^{2}$ of SMSS DR3, which covers nearly all the sky at declination $\delta<+2^{\circ}$; missing parts are primarily found very close to the Galactic plane and the Galactic Centre. In this work, we avoid areas with high object density, where photometry may be challenging, and high reddening, where highredshift QSOs may be dimmed beyond our search depth. Hence, we focus on Galactic latitudes $|b|>15^{\circ}$. These two simple geometric rules define an area that covers 38.35 per cent of the full sky, or $15821 \mathrm{deg}^{2}$

We further exclude specific areas: by default, bright stars in DR3 are surrounded by exclusion zones (for details see Onken et al. 2019), where detected sources are flagged and prevented from inclusion in the master table. For this work, the exclusion zones in $z$-band are relevant, which add up to $134 \mathrm{deg}^{2}$ within the search area. We also exclude areas around nearby galaxies, where, e.g., red supergiants and long-period variable stars can contaminate the QSO candidate list. We use the Updated Nearby Galaxy Catalog (Karachentsev, Makarov, \& Kaisina 2013) of 869 galaxies from the Local Volume, and mask the sky within $1.3 \times$ the major angular diameter $a_{26}$ (which corresponds to the Holmberg isophote of $\sim 26.5 \mathrm{mag}(\mathrm{Vega}) \mathrm{arcsec}^{-2}$ in the $B$ band) of all the galaxies. The largest resulting exclusion zones are around the LMC and SMC with 6.99 and 4.12 deg radius, respectively, as well as the region around the core of Sgr dSph that reaches to $|b|>15^{\circ}$. All further galaxies have exclusion zones smaller than $0.5 \mathrm{deg}$, with the Sculptor dwarf galaxy, NGC 55, NGC 253 and Centaurus A being the next largest objects. The total excluded area is $276.5 \mathrm{deg}^{2}$.

We then select objects in the SkyMapper $z$-band, which has an effective mean wavelength of $916 \mathrm{~nm}$ and a FWHM of $84 \mathrm{~nm}$. The bandpass efficiency curve is asymmetric as it rises steeply at the blue edge but rolls off to the red with the declining sensitivity of the CCD detectors (see Bessell et al. 2011). We limit the candidate selection to $z_{\mathrm{PSF}}<19.5 \mathrm{ABmag}$, although source incompleteness sets in at $z_{\text {PSF }}>18.5 \mathrm{ABmag}$, with a dependence on sky location originating from the SMSS progress as of DR3. Magnitude dependence of the incompleteness is discussed in detail below (Sect. 4.2.3).

From this SMSS DR3 list, we then use position-based crossmatches to other large-area surveys, such as those from Gaia, WISE, and VISTA. As new versions of the WISE and Gaia data sets were released in December 2020, we replaced the AllWISE photometry for the $W 1$ and $W 2$ bands with the CatWISE2020 catalogue (Marocco et al. 2021, hereafter CatWISE), which is more precise for non-variable objects; with the release of Gaia eDR3 (Gaia Collaboration et al. 2021), we updated our original selection from Gaia DR2 in order to further reduce our candidate lists with improved parallax and proper motion (PPM) information.

Using cross-matches with Gaia eDR3 contained in the DR3 master table, we consider objects where the nearest Gaia source is within 0.5 arcsec, and the second-nearest is at least 5 arcsec away. Close pairs of sources in Gaia often appear as one single SMSS source, causing the primary match to be more than 0.5 arcsec offset and showing a secondary match within 1 to 2 arcsec. Neighbours within 5 arcsec may affect the WISE photometry, given a WISE $W 1 / W 2$ PSF with $\sim 6$ " FWHM, which would be detrimental to separating QSOs from cool stars. We require that Gaia has PPM data for the source and that the SMSS DR3 $z$-band photometry flags are $<4$ (indicating reliable measurements). We estimate the fractional loss of objects due to these requirements by considering

2 See https://skymapper.anu.edu.au for catalogue details. 
all known QSOs from the Milliquas v7.1 catalogue (Flesch 2015) in our search area that have $z>3$ and $z_{\mathrm{PSF}}=16-19 \mathrm{ABmag}$. We find that we lose 6 per cent of objects, mostly due to the requirement of no neighbour being present within 5 arcsec. At this point, we end up with an effective search area of 35.1 per cent of the full sky, or $14486 \mathrm{deg}^{2}$

Then we take advantage of cross-matches between the SMSS master table and each of AllWISE and CatWISE, using only matches within 2 arcsec. Not all DR3 objects have a counterpart, but all known QSOs at $z>4$ with $z$ PSF $<19$ ABmag do so. Marocco et al. (2021) quote the 90 per cent completeness depth for CatWISE as $W 1=17.7 \mathrm{mag}$ and $W 2=17.5 \mathrm{mag}$. To examine the impact on our QSO search, we estimate the mean expected $z$ band magnitude of $z \sim 5$ QSOs at the $W 2$ completeness limit from the average colour of bright ( $W 2<15 \mathrm{mag}$ ) known $z \sim 5$ QSOs, which is $z \mathrm{PSF}-W 2 \approx 3.75$. Hence, at $W 2=17.5$ mag we expect $z_{\mathrm{PSF}} \approx 21.25 \mathrm{ABmag}$, which exceeds the depth of our planned search by $\sim 2$ mag. Brighter DR3 objects without WISE matches are most likely stars and not QSOs, because cool stars have bluer optical-minus-MIR colours. We also use data from the $W 3$ band where it is available, but we do not require it for selection, because the 5-sigma sensitivity of $W 3=11.3 \mathrm{mag}$ is too shallow for our purposes. Overall, we assume that the WISE data introduces no incompleteness into the high-redshift QSOs selection. In contrast, Y16 did find ALLWISE-imposed incompleteness to be important; however, such constraints only appeared at fainter magnitudes than are relevant for our search $(W 1>17 \mathrm{mag}, W 2>16 \mathrm{mag})$.

We note that about 6 per cent of $z>4$ QSOs from Milliquas v7.1 lack a CatWISE entry, although all of them are present in AllWISE. Thus, we use CatWISE data where available and fill in AllWISE data where needed. As the $W 1-W 2$ colour has been found useful for separating QSOs from cool stars and is also used in this work, we compared this colour for QSOs between AllWISE and CatWISE. We found the quoted uncertainties in $W 1-W 2$ to shrink by a factor of 2.3 and the colour changes per object from AllWISE to CatWISE to be statistically consistent with the errors quoted in AllWISE. We also find that the colour range of high-redshift QSOs shrinks slightly, in line with the quoted error properties, although this test has only moderate significance given the small numbers of objects.

\section{CANDIDATE SELECTION}

In this section, we discuss the properties of known QSOs brighter than $z_{\mathrm{PSF}}=19 \mathrm{ABmag}$ at redshift $4-6$. We include all known QSOs in our search area, as listed in Milliquas v7.1, which includes QSOs published until $14 \mathrm{Feb} 2021^{3}$. Based on the distribution of these known objects, we propose selection criteria for high-redshift QSOs that we assume to be complete except for rare cases with unusual colours. The sample of candidates resulting from these criteria has not been observed completely with spectroscopy but can inform future observations and the completeness of the verified samples.

3 While this paper was under review, new releases of Milliquas (up to v7.3c on 28 Nov 2021) added 24 new QSOs discovered by other groups with confirmed redshifts between 4 and 6, mostly from Wenzl et al. (2021). However, only three are both in our search area and have $z$ PSF $<19$ ABmag; two were unconfirmed at the time of observation and are reported as "discoveries" in our sample, as indicated in Table 3, and the third has $z_{\mathrm{PSF}}=18.9 \mathrm{ABmag}$, at which depth our spectroscopic follow-up remains incomplete.

\subsection{PPM information from Gaia}

Gaia eDR3 provides measurements of the parallax $\pi$, the proper motion $\mu_{\alpha}, \mu_{\delta}$, and their errors $\sigma_{\pi}, \sigma_{\alpha}$ and $\sigma_{\delta}$ for most objects. For QSOs we demand that these are all consistent with zero within the errors. We calculate a $\chi^{2}$-style measure of consistency using

$S N_{\mathrm{PPM}}=\sqrt{\frac{1}{3}\left(\left(\frac{\max (0, \pi)}{\sigma_{\pi}}\right)^{2}+\left(\frac{\mu_{\alpha}}{\sigma_{\alpha}}\right)^{2}+\left(\frac{\mu_{\delta}}{\sigma_{\delta}}\right)^{2}\right)}$

Our survey footprint contains 137 QSOs known before this work at magnitude $z_{\mathrm{PSF}}<19 \mathrm{ABmag}$ and redshift $z \geq 4$. Of these, 132 objects ( 96 per cent) have $S N_{\mathrm{PPM}}<2$ and 88 (64 per cent) have $S N_{\mathrm{PPM}}<1$, in excellent agreement with the statistics expected for a population without parallax and proper motion. We also find no noticeable trend in the PPM properties with magnitude or redshift, which all lends high credibility to the quoted Gaia PPM measurement uncertainties.

\subsection{Photometric information}

Altogether, we consider the following photometric data: from SMSS DR3, the PSF magnitudes in the passbands griz (for full filter information, see Wolf et al. 2018a) as well as photometric flag information; from Gaia eDR3, the passbands $G, B_{p}$, and $R_{p}$, as well as the Bp/Rp Excess Factor; from VHS DR6 and VIKING DR5, the nearinfrared passbands $J H K$; from CatWISE, the mid-infrared passbands $W 1$ and $W 2$; and from AllWISE, the passband $W 3$. We correct the colours for interstellar foreground reddening using the extinction map from Schlegel, Finkbeiner, \& Davis (1998, hereafter SFD) with extinction coefficients for Gaia from Casagrande \& VandenBerg (2018) for use in $A_{\text {band }}=R_{\text {band }} \times 0.86 \times E(B-V)_{\mathrm{SFD}}$ and for SkyMapper from Wolf et al. (2018a) for use in $A_{\text {band }}=R_{\text {band }} \times$ $E(B-V)_{\mathrm{SFD}}$, as the Schlafly \& Finkbeiner (2011) correction factor 0.86 is already absorbed into the extinction coefficients. In the NIR, we had chosen $\left(A_{J}, A_{H}, A_{K}\right)=(0.8,0.5,0.3) \times 0.86 E(B-V)$, which is a little different from the values $(0.75,0.41,0.24)$ suggested by Wang \& Chen (2019), but the effects are mostly on the order of $0.01 \mathrm{mag}$ in the low-reddening areas we are searching. We assume no extinction in the WISE passbands.

First we address an issue with Gaia photometry of faint red sources: in Fig. 1 we show the Bp/Rp Excess Factor and the colours $G-R_{p}$ and $B_{p}-R_{p}$ vs. $G$ magnitude of all QSOs known in the search area within a narrow redshift range of $4.7<z<5$.3. While there is no trend of $G-R_{p}$ colour with brightness in $G$, there is a strong trend of $B_{p}-R_{p}$ getting bluer and the Excess Factor increasing as the magnitude gets fainter. Riello et al. (2021) discuss the origin of this bias towards higher $B_{p}$ fluxes in red objects that are near the detection limit in the $B_{p}$ channel. Here, we attempt to rectify the $B_{p}-R_{p}$ colour with an ad-hoc quadratic correction, only for objects with $G>19 \mathrm{mag}$, of the form

$B_{p, \mathrm{c}}-R_{p}=B_{p}-R_{p}+0.3 \times(\max (0, G-19))^{2}$.

The right panel in the figure shows the result of this correction, and in the following we adopt such corrected $B_{p, \mathrm{c}}-R_{p}$ colours.

Figure 2 shows the redshift trends of several colour indices formed from the passband measurements and corrected for foreground reddening. We see well-known features:

(i) Strong trends of colour with redshift arise as the spectral step from the unabsorbed QSO continuum to the Ly $\alpha$ forest near 

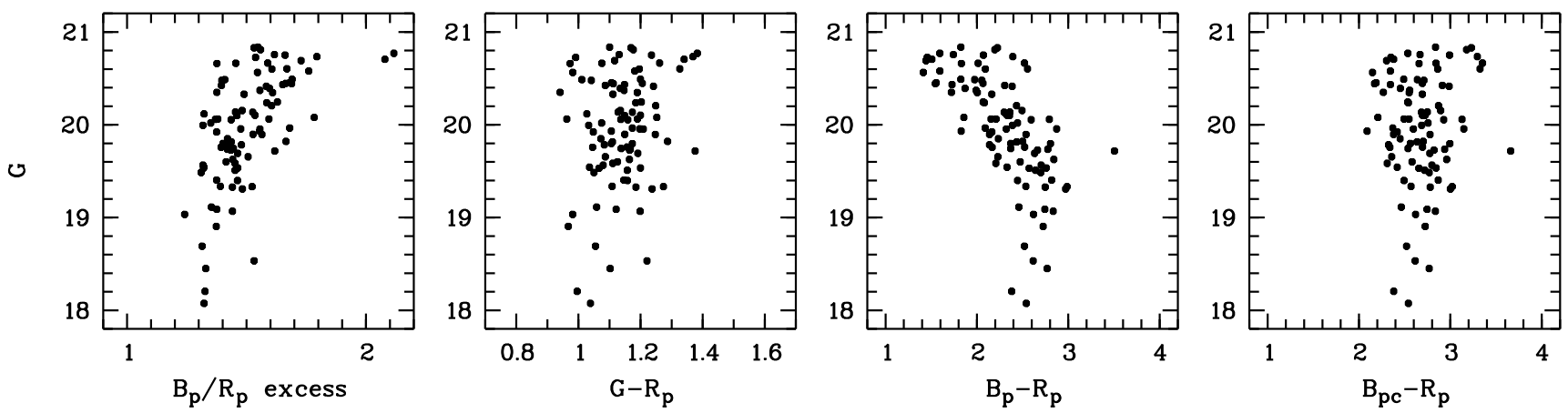

Figure 1. Gaia photometry of redshift $4.7<z<5.3$ QSOs near the faint end, where little flux is expected in the $B_{p}$ channel, while the $G$ band and the $R_{p}$ channel should still see significant flux: the Bp/Rp Excess Factor increases from a value of $\sim 1.3$, which is typical for point sources of this colour, to values around 2 near the detection limit (left panel); the $G-R_{p}$ colour shows no trend with magnitude (centre-left), but the $B_{p}-R_{p}$ colour drifts bluewards toward the detection limit (centre-right), suggesting extra flux being recorded. We use the ad-hoc quadratic correction from Eq. 2 to rectify the $B_{p}$ bias (right panel).

$\lambda_{\text {rest }} \simeq 120 \mathrm{~nm}$ is redshifted through the spectral range, see e.g. $G-R_{p}, B_{p, \mathrm{c}}-R_{p}, G-r_{\mathrm{PSF}}, g_{\mathrm{PSF}}-r_{\mathrm{PSF}}$, and $r_{\mathrm{PSF}}-i_{\mathrm{PSF}}$.

(ii) Where both passbands in a colour index probe unabsorbed continuum, it reflects the spectral slope of the continuum, which has only moderate scatter in the bulk of the QSO population, see e.g. $z \mathrm{PSF}-J, J-K$ and $K-W 1$.

(iii) Emission lines vary colours slightly as they are redshifted through the passbands, most notably the strong line $\mathrm{H} \alpha$ in the colours $K-W 1$ and $W 1-W 2$, where variations with redshift trace the structured passband efficiency curve of $W 1$.

\subsection{QSO selection criteria}

Based on the distribution of colours observed for the existing QSO sample, we propose the following set of selection criteria to define the volume of QSO candidates at $z \geq 4.4$ :

$$
\begin{aligned}
& 0.8<G-R_{p}<1.8 \\
& 1.8<B_{p, \mathrm{c}}-R_{p} \\
& 0.9<g_{\mathrm{PSF}}-r_{\mathrm{PSF}} \\
& 0.7<J-K<1.8 \\
& 1.5<J-W 1<3 \\
& 0.2<W 1-W 2<1.1 \\
& 2.3<W 1-W 3<4.7 \text { if measured }
\end{aligned}
$$

We further define three indices, $i_{1}, i_{2}$ and $i_{3}$, to reduce the contamination from broad absorption line QSOs (BALQSOs) at $z<4$ and from distant cool stars:

$$
\begin{array}{llcl}
0 & < & (J-K)+\left(B_{p, \mathrm{c}}-R_{p}\right)-\left(z_{\mathrm{PSF}}-J\right)-1.8 & =i_{1} \\
0< & (J-W 1)-1.4\left(z_{\mathrm{PSF}}-J\right)+0.1 & =i_{2} \\
0< & 0.6-0.5\left(r_{\mathrm{PSF}}-i_{\mathrm{PSF}}\right)-\left(G-r_{\mathrm{PSF}}\right) & =i_{3}
\end{array}
$$

We include objects that are undetected in $r$-band. Considering the colour trends with redshift, it would seem reasonable to make stronger use of the $r_{\mathrm{PSF}}-i_{\mathrm{PSF}}$ colour. Unfortunately, this requires $r$-band data at the depth of the SMSS Main Survey, but in DR3 the hemisphere is only complete with deep data in $i$-band and $z$-band, while deep $r$-band covers only two thirds of the hemisphere. A nondetection in $r$-band may thus be either a sign of high redshift or a sign of shallow data, depending on the sky position. Hence, we do not attempt to fully exploit the $r$-band information at this stage. Finally, we apply a cut in PPM significance (see Eq. 1):

$S N_{\mathrm{PPM}}<2$, which should make us lose only around 4 per cent of the QSO sample, while keeping contamination by Milky Way stars low.

These simple geometric selection rules may seem suboptimal, as several authors have convincingly argued that other techniques - a general Bayesian selection (e.g. Wolf, Meisenheimer, \& Röser 2001; Richards et al. 2009; Mortlock et al. 2012; Reed et al. 2017), or machine learning approaches (e.g., Calderone et al. 2019; Wenzl et al. 2021; Guarneri et al. 2021) - ought to be superior in modelling the amorphous locus of QSOs in high-dimensional SED space. However, previous QSO samples at high redshift were incomplete, with a highly structured selection function, which we do not want to propagate into our study. After all, this work aims at improving the completeness of observed samples. Hence, we chose not to apply elaborate statistical methods to inferior training samples when informing our follow-up observations.

The final candidate list within our selection volume includes 197 objects brighter than $z_{\mathrm{PSF}}=18.7 \mathrm{ABmag}$. Of these, 64 were known QSOs as of Milliquas v7.1, with 17 reported by (Wolf et al. 2018b) and Paper I, and a further 47 reported by a variety of authors including W16, Yang et al. (2017), Schindler et al. (2019a), Yang et al. (2019), and Lyke et al. (2020). This left 133 new candidates with $z_{\mathrm{PSF}}<18.7 \mathrm{ABmag}$ to be followed up. The list includes a further 497 fainter candidates with $z_{\mathrm{PSF}}>18.7 \mathrm{ABmag}$, of which 21 were known QSOs as of Milliquas v7.1, leaving 476 candidates to follow up in this and future work.

\section{SPECTROSCOPY AT THE ANU 2.3M TELESCOPE: 2017 TO 2021}

This work builds on the study of Paper I and extends it to fainter magnitudes from SMSS DR3. We have followed up 132 of the 133 unstudied objects from our $z \mathrm{PSF}<18.7 \mathrm{ABmag}$ candidate list. Also, we followed up 154 fainter objects that extend to $z_{\mathrm{PSF}} \simeq 19 \mathrm{ABmag}$, about 1 mag deeper than Paper I.

We note that during the early phase of this work, we had not yet applied the faint correction to the Gaia $B_{p}$ magnitudes mentioned in Sect. 3.2. At that stage, we had additional candidates with $B_{p}-R_{p}>1.8$ mag but $B_{p, c}-R_{p}<1.8 \mathrm{mag}$, a small number of which we have followed up, as shown by the black points outside the selection cut in the $B_{p, c}-R_{p}$ panel of Fig. 4. These objects, however, did not end up in our complete sample discussed later, as they were all in the incomplete redshift range at $z<4.4$.

In addition, we have pursued a complementary search by occasionally sampling objects outside our selection criteria, in order 

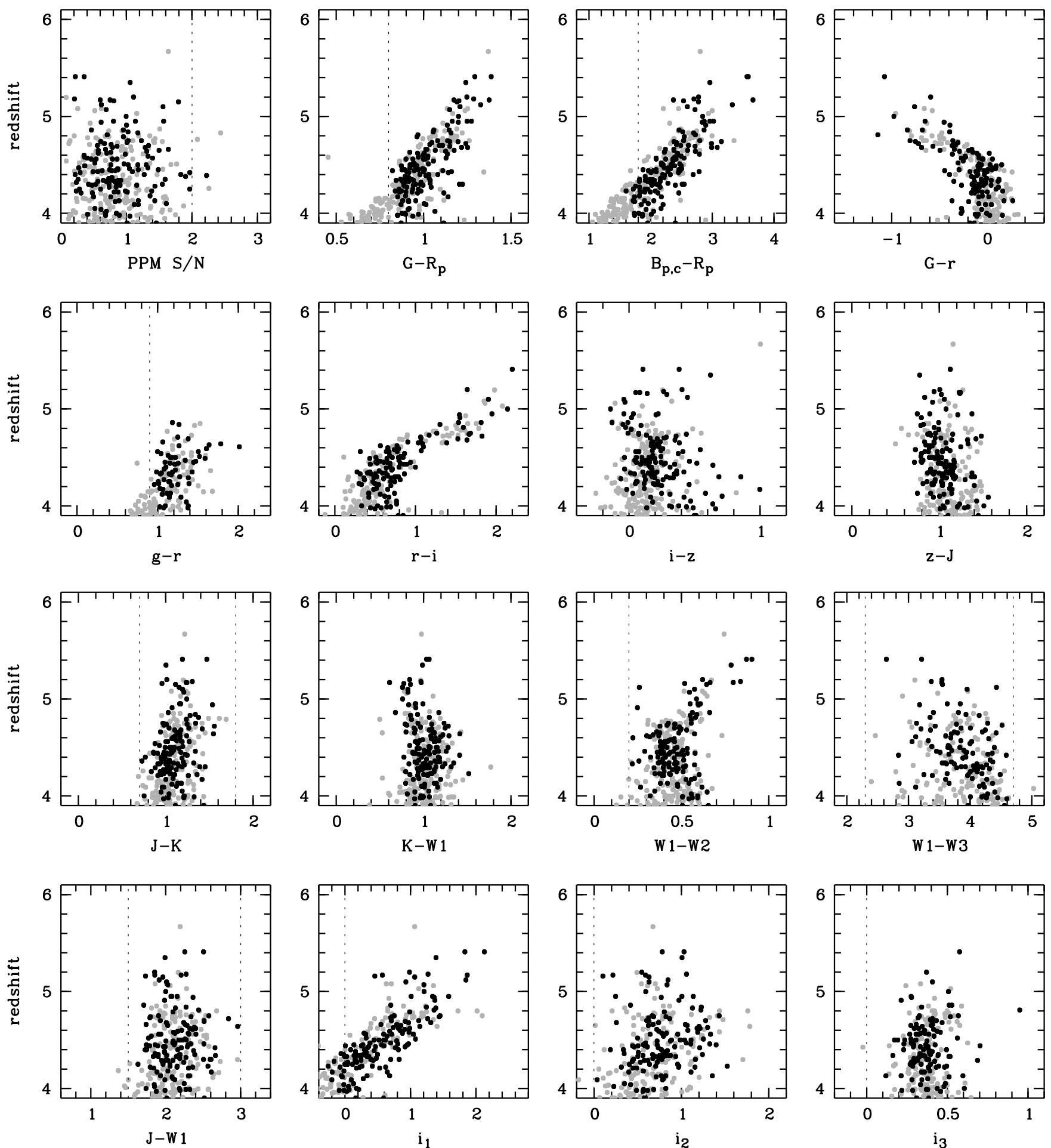

Figure 2. Characteristics of known high-redshift QSOs with $z \mathrm{PSF}<19 \mathrm{ABmag}$ at redshift $z>3.9$ : significance of parallax and proper motion signal (top left) and colour indices (all other panels). Grey points are previously known QSOs and black points are new objects presented in this work. Dashed lines indicate the adopted selection criteria, which eliminate only four of the QSOs at $z \geq 4$.4. The colour indices $i_{1}, i_{2}$, and $i_{3}$ are defined in Eq. 4. A few of the $z<4.4$ QSOs discovered in this work were inside the selection criteria before we corrected the faint Gaia $B_{p}$ magnitudes as explained in Sect. 3.2. 

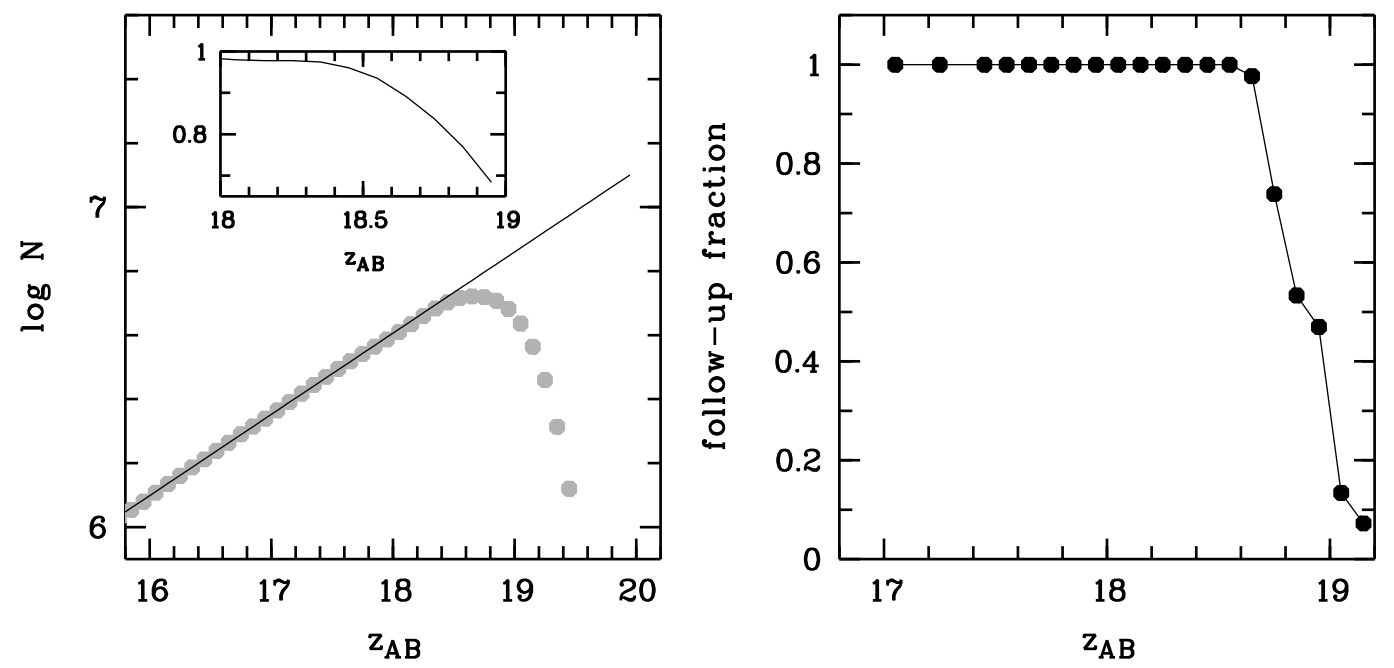

Figure 3. Completeness diagnostics: the SMSS $z$-band source list is incomplete at $z_{\mathrm{PSF}}>18.5 \mathrm{ABmag}$ (left panel), as diagnosed by the source number counts in the search area; the derived source completeness as shown in the inset declines from 95 per cent at $z$ PSF $=18.5 \mathrm{ABmag}$ to 63 per cent at $z$ PSF $=19 \mathrm{ABmag}$. Starting from the source list, the spectroscopic follow-up is complete at $z_{\mathrm{PSF}}<18.7 \mathrm{ABmag}$, while completeness drops to $\sim 10$ per cent at $z \mathrm{PSF}=19 \mathrm{ABmag}$.

to explore whether we are missing an unknown QSO population at high redshift. However, the only high-redshift QSO discovered this way was one at $z=4.39$, whose colours were indeed all within the selection criteria, while its PPM signal was slightly larger than our cutoff.

We have used the Wide Field Spectrograph (WiFeS; Dopita et al. 2010) on the ANU 2.3m telescope at Siding Spring Observatory for a number of nights between December 2017 and October 2021. In conjunction with the standard RT560 beam-splitter, we primarily used the $\mathrm{WiFeS} \mathrm{B} 3000$ and $\mathrm{R} 3000$ gratings in the blue and red arm, respectively, which together cover the wavelength range from $360 \mathrm{~nm}$ to $980 \mathrm{~nm}$ at a resolution of $R=3000$. This setup allows us to see QSO spectra from the Ly $\alpha$ forest to the C IV line for all objects at redshift $z<5$. At redshift $z>5$, the $\mathrm{C}$ Iv line starts to get lost in the sky noise and for the highest-redshift objects it is even outside the data range. However, in these cases the Ly $\alpha$ forest, in combination with the Ly $\alpha$ and Si Iv line, are sufficient to confirm objects as high-redshift QSOs. Because of mechanical issues with the instrument, two nights used alternative gratings in the red arm: I7000 in December 2020 and R7000 in April 2021. Despite the restricted wavelength range in each case, numerous candidates were successfully classified. Exposure times ranged from $600 \mathrm{sec}$ to $2400 \mathrm{sec}$, and observing conditions varied in terms of cloud cover and seeing. For the faintest objects, we obtained and co-added two spectra of up to $2400 \mathrm{sec}$ each.

The data were reduced using the Python-based pipeline PyWiFeS (Childress et al. 2014). PyWiFeS calibrates the raw data with bias, arc, wire, internal-flat and sky-flat frames, and performs flux calibration and telluric correction with standard star spectra. Flux densities were calibrated using a number of standard stars, which are usually observed on the same night, although not necessarily under the same cloud conditions. ${ }^{4}$ We then extracted spectra from the calibrated 3D data cube using QFitsView ${ }^{5}$. Reduced spectra were visualised with the MARZ software (Hinton et al. 2016) and template spectra were overplotted to aid the classification and

\footnotetext{
4 This lack of robust flux calibration precludes an independent estimation of the absolute magnitudes from the spectroscopy.

5 https://www.mpe.mpg.de/ ott/QFitsView/
}

redshift determination. We estimate redshifts from broad $\mathrm{Si}$ IV, $\mathrm{N} \mathrm{v}$, and $C_{\text {IV }}$ lines where available, and consider the blue edge of the Ly $\alpha$ line when necessary. Because of frequent absorption within the emission line profiles and the possibility of $\mathrm{C}$ Iv blueshifts, we determined the redshifts manually, and thus we estimate that the redshift uncertainties range from 0.01 to $\sim 0.05$ for weak-lined objects.

Since December 2017, spectra were taken for 739 objects, of which 577 provided sufficient signal for a confident classification. Most of the targets are not candidates anymore after using the refined PPM data of Gaia eDR3. 252 targets were observed to be QSOs, 67 per cent of which are at $z>3.8$. (Among those 170 QSOs were seven discovered by other groups and mistakenly included in our observing lists.) QSOs at lower redshift include objects with red continua as well as extreme BALQSOs and overlapping iron trough low-ionisation BALQSOs (OFeLoBALQSOs), which mimic the optical colours of high-redshift QSOs, but have MIR colours typical of their redshift, hence we have learned how they can largely be avoided from the start. Several stars contaminated the candidate lists, especially before the Gaia eDR3 release. Altogether 13 stars turned out to be likely red supergiants in nearby galaxies including NGC 300, the Sculptor and Fornax dwarf galaxies as well as the SMC and LMC and later we chose to exclude the sky areas covered by these.

\subsection{Newly identified high-redshift QSOs}

Among the 197 candidates defined by the final selection criteria in the nearly complete magnitude range at $z_{\mathrm{PSF}}<18.7 \mathrm{ABmag}$, there are $116 z \geq 4$ QSOs, 29 (mostly BAL) QSOs at $z<4$ and 19 stars. The remaining objects are not high-redshift QSOs, although we cannot type them confidently, either due to low signal in the spectra or due to unusual spectral features. As described in Sect. 4.2.1, only 4 known high-redshift QSOs were excluded by these criteria.

In Tables 1-3, we report our 142 newly identified high-redshift QSOs, split into three redshift ranges. The highest-redshift objects are at $z=5.41$, and together with the objects from Paper I, the total number of $z>3.8$ QSOs discovered with this project is 163 , of which 90 per cent have $M_{145}<-27$ ABmag.

The tables list $z$-band $\mathrm{AB}$ and $H$-band Vega magnitudes, which 

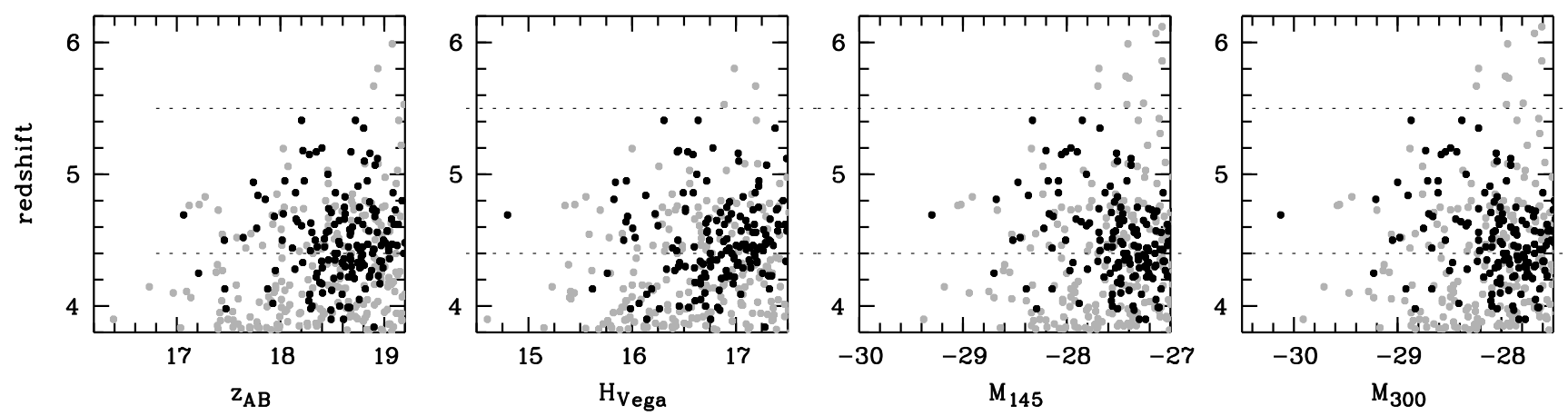

Figure 4. The current Southern bright high-redshift QSO sample: apparent optical and IR magnitude (left and centre-left panels) and luminosity at 145 nm and $300 \mathrm{~nm}$ (centre-right and right). Dark points are QSOs found with SkyMapper and reported by Wolf et al. (2018b), Paper I, and this work. Light points are QSOs from the literature in the search area targeted in this work. The relative lack of objects at redshift $z>5.5$ suggests the sample is incomplete there.

are useful for the LF construction and for planning more detailed follow-up observations. ${ }^{6}$ In many cases, the $H$-band magnitude is not observed but inferred from $J$ and $K$ magnitudes, or if necessary, from the $z \mathrm{PSF}$ and $W 1$ photometry. We use the mean colour relations that we find in the sample, which are $H-K=0.41(J-K)$ and $H-W 1=-0.007+0.4677\left(z_{\mathrm{PSF}}-W 1\right)$. The relations are roughly linear across colour, with a root mean square (RMS) scatter of $0.1 \mathrm{mag}$ and $0.15 \mathrm{mag}$, respectively, and have no trend with redshift in our range of concern.

\subsection{Completeness considerations}

In this section, we explore how to define a complete sample for use in the luminosity functions. Our selection criteria were adopted to provide a complete sample of QSOs at redshift $z \geq 4.4$, at least to a depth of $z_{\mathrm{PSF}} \approx 19 \mathrm{ABmag}$. At fainter magnitudes, the uncertainties in colours broaden the QSO distribution and the significance of the PPM information degrades, making our approach less effective.

\subsubsection{Colour and PPM selection effects}

Currently, there are 143 QSOs known in our search area with $z \geq 4.4$ and $z_{\mathrm{PSF}}<19 \mathrm{ABmag}$. Here, we have assumed that all literature QSOs within these limits are contained in the compilation Milliquas v7.3 (Flesch 2015). Of the known QSOs, four objects are rejected by the selection criteria of Sect. 3.3: two because of the PPM signal and two more because of their colours. One additional known $z=4.87$ QSO is nearly blended with a cool star and thus eliminated by our requirement for no close neighbours, which has reduced our effective search area. Hence, we conclude that our PPM and colour selection criteria may miss $\sim 3$ per cent of the candidates across our search area of $14,486 \mathrm{deg}^{2}$.

While considering the known QSOs implies that we inherit traditional selection biases, we note that we were able to relax and extend the selection beyond previous rules, because the Gaia PPM data vastly reduced the stellar contamination. The majority of objects in the sample considered here are new QSOs identified using our extended cuts. By considering our selection boundaries relative

\footnotetext{
6 One faint source in Table 2 has no $z$-band photometry in SMSS DR3, and here we substituted the photometry from the NOIRLab Source Catalog (NSC) DR2 (Nidever et al. 2021). We have been unable to locate any NIR photometry for the source, but the lack of an $M_{300}$ estimate does not affect our analysis, because it falls well below the brightness regime in which we are complete and from which we construct the LFs.
}

to the shape of the QSO distributions against colour in Fig. 2, we see that we capture not only the bulk of the QSO population but also its tails, and that the remaining incompleteness based on the photometric selection cuts must be small.

\subsubsection{Upper redshift limit?}

While we make no attempt to avoid the detection of QSOs at the highest possible redshifts, we assume that our search is only complete to $z \simeq 5.4$, where we find our two highest-redshift objects. Inspecting Fig. 4 suggests that the sample is conspicuously devoid of bright QSOs at $z>5.5$.

Here, it is worth highlighting our reliance on Gaia data and the redshift constraints this imposes. Beyond redshift $\sim 5.5$, very little flux remains in the Gaia $G$ passband from which the astrometric measurements are made. In fact, none of the known QSOs beyond $\mathrm{z}=5.7$ have Gaia parallax measurements in eDR3. In the regime between $z=5.4$ and $z=5.7$, we expect the Ly $\alpha$ equivalent width to play an important role in determining whether Gaia can measure a parallax or proper motion.

\subsubsection{Incompleteness at the faint end}

A significant consideration in this work is sample incompleteness at the faint end due to two different reasons: first, there is the source incompleteness of SMSS DR3, which we use as a parent sample. We use the $z$-band number counts of objects listed in our search area of DR3 and obtain a linear fit to the brightness range of $z_{\mathrm{PSF}}=$ 16-19 ABmag. The downturn from the linear fit (with slope $0.254 \pm$ 0.001 ) indicates the magnitude-dependent source incompleteness of the parent sample (see left panel in Fig. 3). We find that the completeness declines from 100 per cent at $z_{\mathrm{PSF}}=17 \mathrm{ABmag}$ to 95 per cent at $z_{\mathrm{PSF}}=18.5 \mathrm{ABmag}$, to 86 per cent at $z_{\mathrm{PSF}}=$ 18.7 ABmag, and to 63 per cent at $z_{\mathrm{PSF}}=19 \mathrm{ABmag}$. Secondly, the spectroscopic follow-up of the selected candidate list by the literature and by this work is only complete (bar one candidate) to $z_{\mathrm{PSF}}<18.7 \mathrm{ABmag}$; fainter than that, the completeness drops quickly to $\sim 30$ per cent at $z$ PSF $\simeq 19 \mathrm{ABmag}$.

Secondly, we use NIR photometry from the three surveys 2MASS, VHS and VIKING, with a 2 arcsec matching radius. We find that 6 per cent of Milliquas QSOs with $z>3$ and $z_{\mathrm{PSF}}=16-19$ ABmag have no $J K$ data, which we showed in Sect. 3.3 as being helpful in selecting high-redshift QSOs. This affects mostly fainter QSOs without 2MASS counterparts, in areas where neither the deeper VHS nor the VIKING survey is available 
Table 1. List of newly discovered QSOs. Redshift $3.8 \leq z<4.4$; these are not part of a complete sample and are hence not used for the luminosity functions. Epoch is the date of the first spectrum taken by us. Comments refer to non-standard observing setups or unusual properties. See Appendix A for the spectra.

\begin{tabular}{|c|c|c|c|c|c|c|c|c|c|c|}
\hline SMSS ID & Redshift & $\begin{array}{c}R_{p} \\
\text { (Vega) }\end{array}$ & $\begin{array}{l}z_{\mathrm{PSF}} \\
(\mathrm{AB})\end{array}$ & $\begin{array}{c}H \\
\text { (Vega) }\end{array}$ & $\begin{array}{c}W 1 \\
(\text { Vega) }\end{array}$ & $\begin{array}{c}W 1-W 2 \\
(\text { Vega })\end{array}$ & $\begin{array}{l}M_{145} \\
(\mathrm{AB})\end{array}$ & $\begin{array}{l}M_{300} \\
(\mathrm{AB})\end{array}$ & $\begin{array}{c}\text { Epoch } \\
\text { (spectrum) }\end{array}$ & Comments \\
\hline J000948.16-371708.1 & 4.27 & 17.679 & 17.95 & $16.41 \pm 0.04$ & 15.02 & 0.45 & -27.97 & -28.31 & 20201208 & \\
\hline J003222.03-035807.5 & 4.12 & 18.318 & 18.49 & $16.84 \pm 0.04$ & 15.30 & 0.48 & -27.40 & -27.83 & 20201209 & $\mathrm{I} 7000$ \\
\hline J005938.27-422704.1 & 4.25 & 18.463 & 18.69 & $17.26 \pm 0.03$ & 15.77 & 0.51 & -27.21 & -27.45 & 20201208 & \\
\hline J014615.01-635639.2 & 4.28 & 17.695 & 18.14 & $16.33 \pm 0.04$ & 14.96 & 0.48 & -27.80 & -28.39 & 20200926 & \\
\hline J021218.22-394548.8 & 4.13 & 17.222 & 17.46 & $15.62 \pm 0.03$ & 13.99 & 0.38 & -28.39 & -29.04 & 20201209 & $\mathrm{I} 7000$ \\
\hline J021638.50-033737.8 & 4.31 & 18.546 & 18.81 & $17.01 \pm 0.04$ & 15.44 & 0.52 & -27.14 & -27.72 & 20201001 & \\
\hline J022654.20-322924.0 & 4.30 & 18.973 & 18.78 & $16.51 \pm 0.01$ & 14.80 & 0.52 & -27.15 & -28.21 & 20210118 & \\
\hline J024535.48-445433.0 & 4.02 & 18.027 & 17.93 & $16.07 \pm 0.03$ & 14.82 & 0.56 & -27.88 & -28.55 & 20190703 & \\
\hline J030427.83-780559.2 & 4.00 & 18.400 & 18.57 & $16.71 \pm 0.05$ & 15.18 & 0.59 & -27.36 & -27.95 & 20210118 & \\
\hline J034132.56-411920.9 & 4.30 & 18.940 & 18.68 & $16.45 \pm 0.04$ & 14.65 & 0.51 & -27.25 & -28.28 & 20200927 & \\
\hline J042529.68-395805.8 & 4.36 & 18.890 & 19.05 & $17.54 \pm 0.08$ & 16.17 & 0.54 & -26.93 & -27.46 & 20200810 & \\
\hline J045459.29-420742.6 & 4.17 & 19.398 & 18.96 & $16.97 \pm 0.04$ & 15.19 & 0.56 & -26.91 & -27.70 & 20211022 & \\
\hline J045623.58-085248.5 & 3.90 & 18.676 & 18.49 & $16.14 \pm 0.04$ & 14.44 & 0.65 & -27.30 & -28.44 & 20191130 & \\
\hline J045639.34-402753.6 & 4.18 & 18.087 & 18.38 & $16.61 \pm 0.04$ & 15.21 & 0.57 & -27.51 & -28.08 & 20201209 & $\mathrm{I} 7000$ \\
\hline J052439.12-304800.4 & 4.17 & 18.052 & 18.40 & $16.66 \pm 0.05$ & 15.36 & 0.53 & -27.48 & -28.02 & 20201209 & $\mathrm{I} 7000$ \\
\hline J055901.33-855138.3 & 3.90 & 18.986 & 18.61 & $16.76 \pm 0.06$ & 15.35 & 0.51 & -27.24 & -27.85 & 20200927 & \\
\hline J061751.60-240610.8 & 4.09 & 18.413 & 18.59 & $17.05 \pm 0.10$ & 15.61 & 0.62 & -27.28 & -27.61 & 20210118 & \\
\hline J083905.36-222215.9 & 4.30 & 18.423 & 18.51 & $16.63 \pm 0.05$ & 15.20 & 0.43 & -27.52 & -28.13 & 20200227 & $b_{\mathrm{gal}}=11.5$ \\
\hline J091926.16-094920.5 & 4.38 & 18.338 & 18.67 & $17.18 \pm 0.07$ & 15.74 & 0.39 & -27.31 & -27.57 & 20210411 & $\mathrm{R} 7000$ \\
\hline J092623.99-261620.9 & 4.35 & 18.453 & 18.46 & $16.77 \pm 0.06$ & 15.04 & 0.47 & -27.61 & -28.01 & 20210306 & \\
\hline J095759.35-230941.8 & 4.32 & 18.451 & 18.60 & $16.68 \pm 0.06$ & 15.11 & 0.57 & -27.39 & -28.06 & 20210405 & \\
\hline J104046.17-294635.5 & 4.33 & 18.486 & 18.78 & $17.14 \pm 0.09$ & 15.64 & 0.40 & -27.21 & -27.61 & 20210407 & \\
\hline J110144.63-260043.5 & 4.37 & 18.682 & 18.78 & $16.98 \pm 0.06$ & 15.58 & 0.51 & -27.24 & -27.78 & 20210405 & \\
\hline J113649.79-161939.6 & 4.25 & 18.192 & 18.35 & $16.70 \pm 0.05$ & 15.32 & 0.40 & -27.58 & -28.02 & 20210307 & \\
\hline J120828.90-044419.9 & 4.33 & 18.642 & 18.74 & $17.20 \pm 0.06$ & 15.47 & 0.22 & -27.22 & -27.54 & 20210408 & \\
\hline J131656.26-301244.3 & 4.29 & 18.761 & 18.76 & $17.14 \pm 0.09$ & 15.69 & 0.34 & -27.23 & -27.60 & 20210408 & \\
\hline J134633.39-275654.3 & 3.97 & 18.678 & 18.49 & $16.85 \pm 0.07$ & 15.52 & 0.54 & -27.35 & -27.77 & 20210404 & \\
\hline J135729.80-234839.2 & 4.34 & 18.823 & 18.96 & $17.48 \pm 0.08$ & 16.14 & 0.37 & -27.06 & -27.28 & 20200627 & \\
\hline J151045.68-045007.4 & 4.05 & 18.903 & 18.76 & $16.66 \pm 0.04$ & 14.98 & 0.35 & -27.14 & -27.99 & 20210408 & \\
\hline $\mathrm{J} 151904.00-095230.8$ & 4.22 & 18.432 & 18.65 & $16.90 \pm 0.05$ & 15.60 & 0.60 & -27.36 & -27.84 & 20210409 & \\
\hline J155141.82-153448.9 & 4.33 & 18.230 & 18.21 & $16.46 \pm 0.04$ & 15.13 & 0.46 & -27.94 & -28.35 & 20200221 & weak-lined \\
\hline J160013.90-112800.2 & 4.29 & 18.863 & 18.99 & $17.07 \pm 0.07$ & 15.50 & 0.47 & -27.30 & -27.78 & 20210704 & \\
\hline J164147.78-775029.8 & 4.13 & 18.090 & 17.88 & $16.19 \pm 0.04$ & 14.86 & 0.54 & -28.14 & -28.53 & 20200819 & See Appendix B \\
\hline J172847.61-643404.6 & 4.22 & 18.261 & 18.34 & $16.71 \pm 0.05$ & 15.17 & 0.44 & -27.64 & -28.02 & 20200926 & \\
\hline J181348.59-742305.6 & 4.33 & 18.547 & 18.59 & $17.02 \pm 0.08$ & 15.60 & 0.40 & -27.50 & -27.76 & 20200927 & \\
\hline J182616.06-474625.4 & 4.23 & 18.629 & 18.94 & $17.35 \pm 0.11$ & 15.78 & 0.43 & -27.03 & -27.38 & 20210703 & \\
\hline $\mathrm{J} 185854.75-414702.1$ & 4.34 & 18.762 & 18.97 & $16.75 \pm 0.05$ & 14.82 & 0.48 & -27.07 & -28.02 & 20210704 & \\
\hline J193603.72-702734.4 & 4.39 & 18.580 & 18.79 & $16.86 \pm 0.06$ & 15.15 & 0.46 & -27.26 & -27.92 & 20201001 & \\
\hline J194707.16-443732.3 & 4.13 & 18.876 & 18.82 & $17.32 \pm 0.11$ & 15.69 & 0.42 & -27.09 & -27.36 & 20210704 & \\
\hline $\mathrm{J} 195455.50-222254.5$ & 4.30 & 18.611 & 18.62 & $16.76 \pm 0.05$ & 14.88 & 0.52 & -27.50 & -28.04 & 20210411 & \\
\hline J201808.60-390820.6 & 4.10 & 18.701 & 18.40 & $16.70 \pm 0.06$ & 15.24 & 0.48 & -27.48 & -27.96 & 20201001 & \\
\hline J202712.66-245732.8 & 4.34 & 18.959 & 18.96 & $17.20 \pm 0.07$ & 15.75 & 0.37 & -27.06 & -27.55 & 20210711 & \\
\hline J202914.99-015504.0 & 4.23 & 18.630 & 18.86 & $17.31 \pm 0.08$ & 15.30 & 0.38 & -27.20 & -27.45 & 20210711 & extended? \\
\hline J205659.53-513055.1 & 3.84 & 19.262 & 18.90 & $17.28 \pm 0.06$ & 15.73 & 0.53 & -26.80 & -27.26 & 20210711 & \\
\hline J214938.09-805805.1 & 4.22 & 18.987 & 18.86 & $16.99 \pm 0.07$ & 15.35 & 0.39 & -27.23 & -27.78 & 20201002 & \\
\hline $\mathrm{J} 222820.62-422626.3$ & 3.99 & 18.718 & 18.67 & $16.55 \pm 0.04$ & 15.00 & 0.51 & -27.11 & -28.05 & 20210711 & \\
\hline $\mathrm{J} 230318.14-603123.8$ & 4.35 & 18.136 & 18.52 & $16.82 \pm 0.05$ & 15.23 & 0.38 & -27.44 & -27.92 & 20201210 & \\
\hline $\mathrm{J} 230513.15-031958.9$ & 4.35 & 18.494 & 18.68 & $17.13 \pm 0.05$ & 15.77 & 0.39 & -27.31 & -27.62 & 20210711 & \\
\hline J230824.53-453905.6 & 3.99 & 18.640 & 18.65 & $16.83 \pm 0.04$ & 15.16 & 0.51 & -27.12 & -27.77 & 20210711 & \\
\hline J230857.62-500914.9 & 3.98 & 17.372 & 17.47 & $15.98 \pm 0.01$ & 14.41 & 0.59 & -28.29 & -28.61 & 20190904 & \\
\hline $\mathrm{J} 231141.53-142215.7$ & 3.98 & 18.571 & 18.29 & $16.26 \pm 0.04$ & 14.77 & 0.56 & -27.51 & -28.34 & 20210702 & \\
\hline $\mathrm{J} 231500.34-364049.9$ & 4.27 & 18.842 & 18.88 & $16.91 \pm 0.05$ & 15.32 & 0.47 & -27.05 & -27.81 & 20200627 & \\
\hline J232438.44-201956.9 & 4.23 & 18.285 & 18.58 & $16.94 \pm 0.06$ & 15.45 & 0.35 & -27.34 & -27.77 & 20200926 & \\
\hline
\end{tabular}


Table 2. List of newly discovered QSOs. Redshift $4.4 \leq z<4.7$ QSOs. One faint object has a $z$-band magnitude from the NOIRLab Source Catalog (NSC) DR2. See also caption of Table 1.

\begin{tabular}{|c|c|c|c|c|c|c|c|c|c|c|}
\hline SMSS ID & Redshift & $\begin{array}{c}R_{p} \\
\text { (Vega) }\end{array}$ & $\begin{array}{l}z \mathrm{PSF} \\
(\mathrm{AB})\end{array}$ & $\begin{array}{c}H \\
\text { (Vega) }\end{array}$ & $\begin{array}{c}W 1 \\
(\text { Vega })\end{array}$ & $\begin{array}{c}W 1-W 2 \\
(\text { Vega })\end{array}$ & $\begin{array}{l}M_{145} \\
(\mathrm{AB})\end{array}$ & $\begin{array}{l}M_{300} \\
(\mathrm{AB})\end{array}$ & $\begin{array}{c}\text { Epoch } \\
\text { (spectrum) }\end{array}$ & Comments \\
\hline J003438.57-762140.5 & 4.47 & 18.423 & 18.57 & $17.07 \pm 0.07$ & 15.59 & 0.38 & -27.46 & -27.73 & 20200627 & \\
\hline J003656.23-241844.1 & 4.62 & 18.414 & 18.52 & $16.89 \pm 0.04$ & 15.29 & 0.37 & -27.49 & -27.95 & 20191022 & \\
\hline J004022.28-333316.7 & 4.42 & 18.375 & 18.29 & $16.43 \pm 0.01$ & 14.94 & 0.51 & -27.68 & -28.32 & 20201208 & \\
\hline J004541.14-553511.9 & 4.45 & 18.149 & 18.35 & $17.04 \pm 0.05$ & 15.80 & 0.36 & -27.61 & -27.73 & 20201208 & \\
\hline J004917.31-081723.4 & 4.56 & 18.588 & 18.68 & $17.16 \pm 0.07$ & 15.81 & 0.40 & -27.35 & -27.66 & 20200630 & \\
\hline J010255.67-574246.3 & 4.56 & 18.252 & 18.44 & $16.84 \pm 0.05$ & 15.18 & 0.42 & -27.55 & -27.97 & 20200926 & \\
\hline J014423.70-114948.1 & 4.44 & 18.536 & 19.04 & $16.93 \pm 0.11$ & 15.10 & 0.44 & -26.93 & -27.46 & 20210711 & $z$-band from NSC \\
\hline J020348.61-231453.8 & 4.50 & 18.898 & 18.99 & $17.32 \pm 0.06$ & 15.72 & 0.39 & -26.99 & -27.47 & 20200701 & \\
\hline J024422.99-515931.6 & 4.46 & 18.779 & 19.12 & $17.36 \pm 0.06$ & 15.79 & 0.42 & -26.86 & -27.39 & 20200810 & \\
\hline J024622.42-603753.7 & 4.57 & 18.341 & 18.47 & $16.72 \pm 0.04$ & 15.28 & 0.43 & -27.53 & -28.09 & 20191023 & \\
\hline J025445.42-275042.5 & 4.54 & 18.628 & 18.67 & $17.29 \pm 0.02$ & 16.04 & 0.40 & -27.32 & -27.51 & 20211022 & \\
\hline J025629.33-402041.8 & 4.51 & 18.393 & 18.42 & $16.91 \pm 0.04$ & 15.35 & 0.38 & -27.56 & -27.88 & 20191023 & \\
\hline J030333.78-225121.4 & 4.57 & 18.662 & 18.85 & $17.05 \pm 0.05$ & 15.40 & 0.43 & -27.14 & -27.77 & 20200701 & \\
\hline J031139.48-404428.8 & 4.43 & 18.997 & 18.90 & $17.10 \pm 0.06$ & 15.72 & 0.47 & -27.07 & -27.66 & 20200701 & \\
\hline J032233.76-594328.1 & 4.42 & 18.546 & 18.77 & $16.91 \pm 0.06$ & 14.97 & 0.40 & -27.21 & -27.85 & 20191024 & \\
\hline J033951.43-473959.9 & 4.45 & 18.507 & 18.65 & $17.24 \pm 0.04$ & 15.63 & 0.40 & -27.31 & -27.52 & 20191024 & \\
\hline J034029.49-322353.9 & 4.66 & 18.925 & 19.07 & $17.33 \pm 0.06$ & 15.73 & 0.34 & -26.95 & -27.48 & 20200802 & \\
\hline J034342.87-155923.6 & 4.57 & 18.995 & 19.03 & $17.40 \pm 0.07$ & 15.98 & 0.30 & -27.02 & -27.55 & 20200810 & \\
\hline J034831.18-764305.5 & 4.66 & 18.617 & 18.62 & $16.93 \pm 0.06$ & 15.31 & 0.36 & -27.50 & -27.97 & 20200226 & \\
\hline J035647.24-122512.1 & 4.66 & 18.162 & 18.15 & $16.73 \pm 0.06$ & 15.53 & 0.36 & -27.91 & -28.14 & 20200630 & \\
\hline J044514.74-422805.6 & 4.48 & 18.949 & 19.01 & $17.28 \pm 0.07$ & 15.95 & 0.55 & -26.96 & -27.49 & 20200820 & \\
\hline J045926.71-443855.2 & 4.65 & 18.684 & 18.57 & $16.81 \pm 0.04$ & 15.45 & 0.49 & -27.45 & -28.04 & 20200120 & \\
\hline J051047.28-505722.5 & 4.44 & 18.890 & 18.75 & $17.22 \pm 0.06$ & 15.96 & 0.53 & -27.22 & -27.54 & 20210118 & \\
\hline J093037.26-321931.1 & 4.43 & 18.072 & 18.32 & $16.47 \pm 0.04$ & 14.85 & 0.43 & -27.76 & -28.33 & 20201209 & $b_{\text {gal }}=13.7, \mathrm{I} 7000$ \\
\hline J095735.42-263039.4 & 4.49 & 18.381 & 18.56 & $16.88 \pm 0.07$ & 15.43 & 0.46 & -27.48 & -27.92 & 20200621 & \\
\hline J104556.31-073145.9 & 4.47 & 18.684 & 18.84 & $17.09 \pm 0.04$ & 15.51 & 0.51 & -27.15 & -27.69 & 20200226 & \\
\hline J105124.18-114529.8 & 4.43 & 18.769 & 18.90 & $17.21 \pm 0.03$ & 15.83 & 0.49 & -27.10 & -27.56 & 20200227 & \\
\hline J105702.65-231013.7 & 4.50 & 18.325 & 18.33 & $16.75 \pm 0.05$ & 15.15 & 0.47 & -27.68 & -28.05 & 20200130 & \\
\hline J110948.79-222757.7 & 4.49 & 18.922 & 18.99 & $17.33 \pm 0.08$ & 15.95 & 0.53 & -27.02 & -27.46 & 20200621 & \\
\hline J114230.62-082330.6 & 4.46 & 19.145 & 18.97 & $17.00 \pm 0.05$ & 15.33 & 0.45 & -27.03 & -27.78 & 20200228 & \\
\hline $\mathrm{J} 122749.22-300600.1$ & 4.63 & 18.120 & 18.29 & $16.71 \pm 0.06$ & 14.98 & 0.34 & -27.78 & -28.16 & 20210403 & \\
\hline J125559.18-110812.7 & 4.56 & 18.671 & 18.93 & $17.19 \pm 0.05$ & 15.54 & 0.46 & -27.09 & -27.63 & 20200228 & \\
\hline J133821.00-245845.8 & 4.60 & 18.537 & 18.69 & $17.12 \pm 0.07$ & 15.51 & 0.22 & -27.37 & -27.74 & 20200228 & \\
\hline J134930.11-382208.4 & 4.40 & 18.815 & 18.89 & $17.18 \pm 0.09$ & 15.57 & 0.41 & -27.15 & -27.60 & 20200626 & redshift uncertain \\
\hline J145904.70-160326.6 & 4.46 & 18.617 & 18.74 & $17.16 \pm 0.03$ & 15.48 & 0.29 & -27.35 & -27.64 & 20210408 & extended/multiple \\
\hline J152219.16-271543.7 & 4.44 & 17.942 & 18.11 & $16.39 \pm 0.05$ & 15.02 & 0.38 & -28.14 & -28.47 & 20210404 & \\
\hline J181812.74-564925.1 & 4.62 & 19.106 & 19.09 & $17.47 \pm 0.10$ & 15.98 & 0.46 & -27.03 & -27.56 & 20200813 & \\
\hline J192427.57-582750.5 & 4.45 & 18.823 & 18.79 & $17.12 \pm 0.08$ & 15.78 & 0.44 & -27.32 & -27.70 & 20200616 & \\
\hline J210743.01-494803.3 & 4.48 & 19.027 & 19.20 & $17.61 \pm 0.06$ & 15.93 & 0.39 & -26.80 & -27.33 & 20200812 & \\
\hline J212109.58-404824.4 & 4.68 & 18.934 & 18.91 & $17.04 \pm 0.05$ & 15.48 & 0.53 & -27.16 & -27.84 & 20210711 & \\
\hline J212132.33-651825.6 & 4.40 & 19.057 & 19.19 & $17.71 \pm 0.11$ & 16.19 & 0.45 & -26.80 & -27.33 & 20200813 & \\
\hline J212358.26-391345.4 & 4.57 & 19.014 & 19.03 & $17.49 \pm 0.10$ & 16.00 & 0.23 & -26.99 & -27.52 & 20200812 & \\
\hline J220004.31-291438.6 & 4.62 & 18.617 & 18.79 & $17.18 \pm 0.02$ & 15.44 & 0.30 & -27.23 & -27.67 & 20200616 & \\
\hline $\mathrm{J} 221150.10-525343.5$ & 4.40 & 18.323 & 18.52 & $16.96 \pm 0.04$ & 15.67 & 0.32 & -27.45 & -27.79 & 20210702 & \\
\hline $\mathrm{J} 221423.26-142456.0$ & 4.62 & 19.082 & 19.14 & $17.41 \pm 0.09$ & 15.78 & 0.44 & -26.91 & -27.44 & 20200812 & \\
\hline $\mathrm{J} 225551.95-122635.7$ & 4.44 & 18.633 & 18.77 & $17.14 \pm 0.06$ & 15.82 & 0.58 & -27.23 & -27.63 & 20200630 & \\
\hline J231425.23-665057.9 & 4.47 & 19.064 & 19.08 & $17.29 \pm 0.09$ & 15.86 & 0.45 & -26.91 & -27.44 & 20200813 & \\
\hline J231614.93-461027.6 & 4.65 & 18.704 & 18.75 & $17.10 \pm 0.04$ & 15.51 & 0.38 & -27.26 & -27.76 & 20200627 & \\
\hline J232009.94-605703.5 & 4.58 & 18.773 & 18.67 & $17.27 \pm 0.06$ & 15.81 & 0.44 & -27.33 & -27.56 & 20210712 & \\
\hline $\mathrm{J} 232518.43-584301.2$ & 4.40 & 18.476 & 18.55 & $16.75 \pm 0.02$ & 15.63 & 0.36 & -27.42 & -27.99 & 20210119 & \\
\hline $\mathrm{J} 234842.90-214749.0$ & 4.45 & 18.256 & 18.43 & $16.60 \pm 0.05$ & 15.11 & 0.50 & -27.56 & -28.17 & 20200615 & \\
\hline J235834.56-493325.3 & 4.42 & 18.933 & 19.04 & $17.26 \pm 0.03$ & 15.64 & 0.35 & -26.92 & -27.45 & 20200627 & \\
\hline
\end{tabular}

at present. At $z \mathrm{PSF}<18 \mathrm{ABmag}$ this incompleteness amounts to 2 per cent, and reaches 7 per cent by $z_{\mathrm{PSF}}=18.7 \mathrm{ABmag}$ and $\sim 10$ per cent by $z_{\mathrm{PSF}}=19 \mathrm{ABmag}$.

In conclusion, we have a highly complete sample of $z=$ $4.4-5.4$ QSOs at $z_{\mathrm{PSF}}<18.7 \mathrm{ABmag}$ and a substantial further sample down to $z_{\mathrm{PSF}} \simeq 19 \mathrm{ABmag}$. The completeness at $z_{\text {PSF }}>18.7 \mathrm{ABmag}$ is difficult to estimate reliably given the interplay of source incompleteness in the parent sample, incompleteness in spectroscopic follow-up, and the fraction of true QSOs among the candidates, which will likely decline as we go fainter and contamination by non-QSOs in the candidate sample increases. We thus leave 
Table 3. List of newly discovered QSOs. Redshift $4.7 \leq z<5.5$ QSOs. "W21" indicates two QSOs recently reported by Wenzl et al. (2021). See also caption of Table 1.

\begin{tabular}{|c|c|c|c|c|c|c|c|c|c|c|}
\hline SMSS ID & Redshift & $\begin{array}{c}R_{p} \\
\text { (Vega) }\end{array}$ & $\begin{array}{l}z_{\mathrm{PSF}} \\
(\mathrm{AB})\end{array}$ & $\begin{array}{c}H \\
\text { (Vega) }\end{array}$ & $\begin{array}{c}W 1 \\
\text { (Vega) }\end{array}$ & $\begin{array}{c}W 1-W 2 \\
(\text { Vega })\end{array}$ & $\begin{array}{l}M_{145} \\
(\mathrm{AB})\end{array}$ & $\begin{array}{l}M_{300} \\
(\mathrm{AB})\end{array}$ & $\begin{array}{c}\text { Epoch } \\
\text { (spectrum) }\end{array}$ & Comments \\
\hline J012736.71-300649.6 & 4.73 & 18.909 & 19.11 & $17.17 \pm 0.02$ & 15.78 & 0.52 & -26.97 & -27.50 & 20200810 & \\
\hline J012938.99-582942.7 & 4.75 & 18.594 & 18.76 & $17.22 \pm 0.05$ & 15.88 & 0.56 & -27.32 & -27.68 & 20200120 & \\
\hline J014534.68-163950.3 & 4.71 & 18.497 & 18.49 & $16.85 \pm 0.11$ & 15.43 & 0.49 & -27.57 & -28.03 & 20191128 & \\
\hline J020240.11-294314.4 & 4.86 & 18.894 & 19.08 & $17.19 \pm 0.02$ & 15.80 & 0.55 & -27.07 & -27.75 & 20200818 & \\
\hline J020436.66-252315.2 & 4.86 & 18.249 & 18.53 & $16.59 \pm 0.11$ & 14.90 & 0.66 & -27.63 & -28.35 & 20191020 & \\
\hline J021739.31-125025.0 & 4.73 & 18.862 & 18.89 & $17.39 \pm 0.07$ & 16.17 & 0.42 & -27.18 & -27.50 & 20200630 & \\
\hline J022009.01-352745.3 & 5.07 & 18.942 & 18.91 & $17.30 \pm 0.02$ & 15.94 & 0.55 & -27.38 & -27.72 & 20211020 & \\
\hline J023648.56-114733.7 & 5.20 & 18.634 & 18.40 & $16.78 \pm 0.04$ & 15.51 & 0.62 & -27.96 & -28.29 & 20200926 & \\
\hline J024133.93-543853.0 & 4.74 & 18.723 & 18.89 & $17.40 \pm 0.06$ & 16.18 & 0.46 & -27.21 & -27.51 & 20200630 & \\
\hline J031431.12-573152.7 & 5.10 & 18.732 & 18.81 & $17.03 \pm 0.05$ & 15.71 & 0.57 & -27.51 & -28.00 & 20200801 & \\
\hline J032933.94-410056.1 & 4.83 & 18.596 & 18.62 & $17.07 \pm 0.14$ & 15.69 & 0.55 & -27.51 & -27.86 & 20200626 & \\
\hline J033703.05-254831.5 & 5.15 & 18.493 & 18.28 & $16.59 \pm 0.04$ & 15.27 & 0.64 & -28.05 & -28.46 & 20191128 & $\mathrm{z}=5.08 ?$ \\
\hline J040732.95-281031.3 & 4.75 & 18.467 & 18.62 & $16.91 \pm 0.04$ & 15.11 & 0.43 & -27.48 & -27.99 & 20200120 & \\
\hline J050328.89-195623.0 & 5.00 & 18.316 & 18.46 & $16.62 \pm 0.05$ & 15.34 & 0.59 & -27.81 & -28.37 & 20210203 & \\
\hline J050928.30-183435.1 & 4.70 & 18.579 & 18.62 & $16.96 \pm 0.05$ & 15.62 & 0.46 & -27.46 & -27.93 & 20200820 & \\
\hline J082450.79-674241.5 & 5.41 & 18.775 & 18.72 & $16.64 \pm 0.05$ & 14.95 & 0.90 & -27.85 & -28.38 & 20210118 & \\
\hline J084347.76-253155.6 & 4.75 & 18.629 & 18.62 & $16.92 \pm 0.06$ & 15.53 & 0.41 & -27.56 & -28.01 & 20200119 & $b_{\mathrm{gal}}=10.5, \mathrm{~W} 21$ \\
\hline J113522.01-354838.8 & 4.95 & 18.407 & 18.23 & $16.72 \pm 0.05$ & 15.29 & 0.59 & -28.08 & -28.29 & 20190705 & \\
\hline J121921.12-360933.0 & 4.74 & 18.710 & 18.46 & $16.51 \pm 0.05$ & 14.88 & 0.46 & -27.69 & -28.41 & 20210203 & \\
\hline J162551.54-043049.4 & 5.17 & 18.659 & 18.68 & $16.54 \pm 0.04$ & 15.32 & 0.80 & -27.90 & -28.61 & 20210409 & \\
\hline J162758.93-083343.6 & 4.79 & 18.945 & 18.86 & $16.94 \pm 0.05$ & 15.46 & 0.56 & -27.54 & -28.08 & 20210704 & \\
\hline J165333.86-761426.1 & 5.41 & 18.423 & 18.20 & $16.31 \pm 0.04$ & 14.72 & 0.87 & -28.33 & -28.87 & 20200227 & \\
\hline J192600.92-314202.6 & 5.35 & 19.237 & 18.80 & $17.38 \pm 0.08$ & 15.95 & 0.78 & -27.68 & -27.79 & 20210703 & \\
\hline J194124.59-450023.6 & 5.18 & 18.488 & 18.22 & $16.45 \pm 0.05$ & 14.91 & 0.84 & -28.20 & -28.64 & 20200615 & \\
\hline J205559.20-601147.3 & 4.95 & 18.926 & 18.84 & $17.22 \pm 0.05$ & 15.73 & 0.55 & -27.42 & -27.76 & 20200616 & \\
\hline $\mathrm{J} 211002.60-454548.3$ & 4.80 & 18.878 & 19.17 & $17.46 \pm 0.03$ & 16.16 & 0.57 & -26.96 & -27.49 & 20200813 & \\
\hline J214608.20-485819.5 & 5.17 & 18.325 & 18.34 & $16.44 \pm 0.02$ & 14.88 & 0.66 & -28.01 & -28.63 & 20210429 & \\
\hline
\end{tabular}

the faint-end incompleteness untreated in this work; further followup is progressing to find the missing QSOs at $z_{\mathrm{PSF}}>18.7 \mathrm{ABmag}$.

Cumulative number counts as a function of apparent magnitude ( $z$ - and $H$-band), split into two redshift ranges $(4.4 \leq z<4.7$ and $4.7 \leq z<5.4$; the latter selected to match that of Y16), are shown in the left-hand panels of Fig. 5.

\subsection{Sample comparisons between North and South}

We now want to ask whether the new sample in the Southern hemisphere and the established literature sample in the Northern hemisphere are statistically consistent. Therefore, we look specifically at the redshift range $4.7<z<5.4$ in our sample and compare it with the W16/Y16 sample in the same redshift range that was searched in a Northern area of similar size (within 1 per cent). The comparison between the cumulative number counts for this redshift range is shown in Fig. 5(E). Our sample is $>80$ per cent complete to $z_{\mathrm{PSF}} \simeq 18.7 \mathrm{ABmag}$, where it contains 38 objects, compared to 25 objects in the Y16 sample (they have another 21 objects down to their spectroscopic completeness limit of $z_{\mathrm{SDSS}}=19 \mathrm{ABmag}$ ). This factor of $\sim 1.5$ is easily explained by the different completeness of candidate selection: calculations by Y16 suggest that their candidate selection is 50 to 60 per cent complete, while we assume $>80$ per cent completeness for our sample. In contrast, the slight difference in the $z$ bandpasses of SDSS and SkyMapper plays very little role: comparing the SDSS and SMSS $z$-band AB magnitudes for eight objects in common between Y16 and our Southern sample at $z_{\mathrm{PSF}}<18.7 \mathrm{ABmag}$, we find a mean $z_{\text {SDSS }}-z_{\text {SMSS }}=-0.01 \mathrm{mag}$ with an RMS of 0.14 mag.

Next, we ask whether the completeness of Y16 appears wellestimated in hindsight: we try to apply the selection cuts of Y16 to our sample and count what fraction of objects we lose. The largest difference in the selection is that $\mathrm{Y} 16$ require $W 1-W 2>0.5 \mathrm{mag}$, which was needed in the pre-Gaia era to combat the vast stellar contamination, while we relaxed the cut to $W 1-W 2>0.2$ mag given Gaia's PPM data. At $z$ PSF $<19$ ABmag, our inclusive selection rules revealed 55 QSOs, while using $W 1-W 2>0.5$ mag retains 34 of these (62 per cent), in agreement with the original completeness correction used by Y16. Clearly, there are ultra-luminous $z \sim 5$ QSOs waiting to be discovered in the Northern hemisphere using updated WISE colour cuts and Gaia data.

Among the QSOs selected by our criteria, there are 12 in the SDSS footprint, for which we can fully replicate the Y16 selection rules. We find only one object that the Y16 $W 1-W 2$ colour cut would have retained, but which the other Y16 rules would exclude (SDSS J013127.34-032100.1). Although based on only one object, this suggests a possible, modest $\sim 10$ per cent correction to the Y16 completeness, in addition to the WISE-based factor above. 

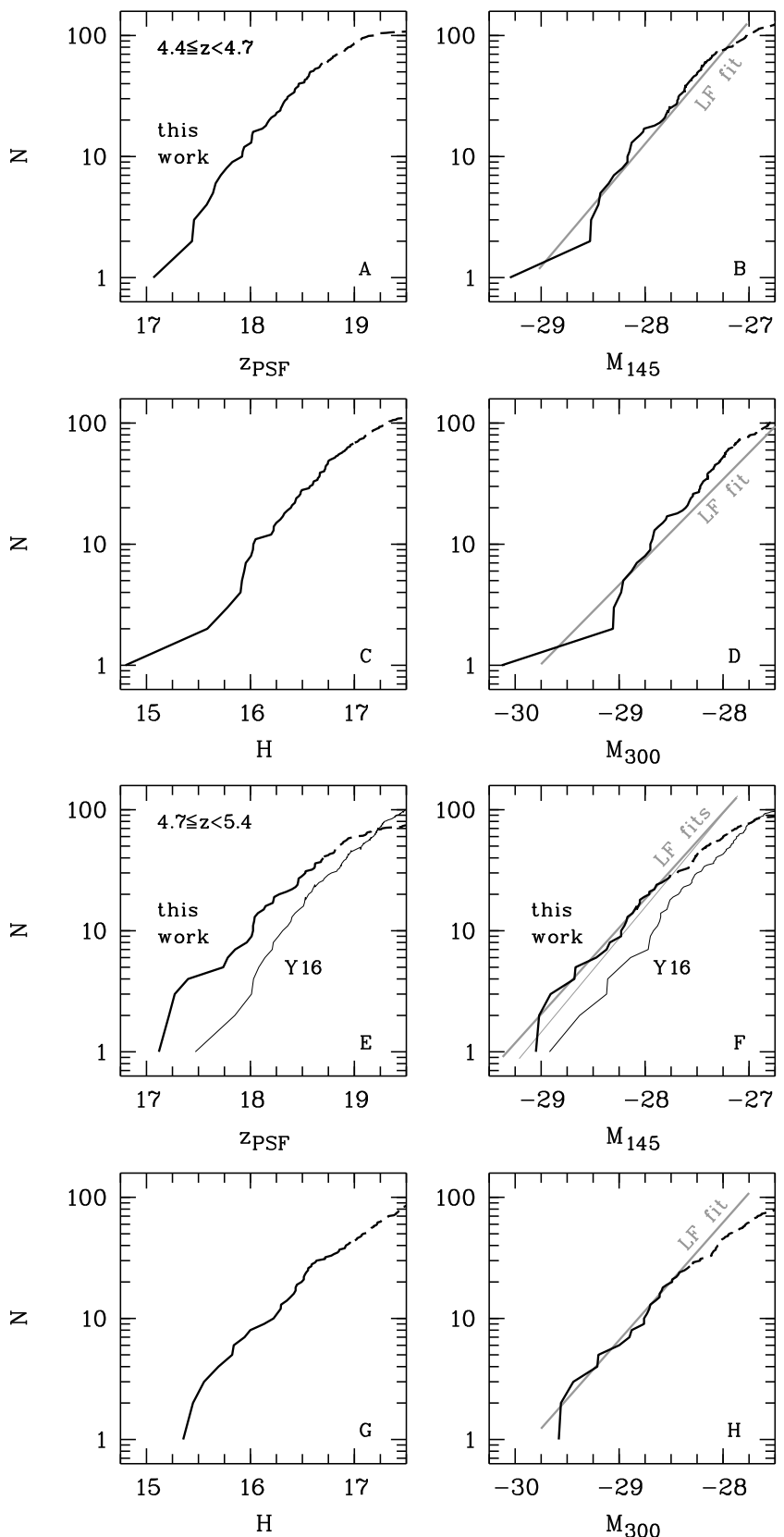

Figure 5. Cumulative QSO number counts vs. apparent magnitude in $z$ - and $H$-band (panels ACEG) and vs. absolute magnitude $M_{145}$ and $M_{300}$ (panels $\mathrm{BDFH}$ ). The top 4 panels (ABCD) show $4.4 \leq z<4.7$, while the bottom 4 panels $(\mathrm{EFGH})$ show $4.7 \leq z<5.4$. The incomplete tail of our sample line is dashed. Panels E and F include a comparison with the Y16 sample. Thick grey lines are predictions from our luminosity function fits (the thin grey line in panel F is the Y16 fit, renormalised to our cosmology and search area).

\subsection{Estimation of $M_{145}$ and $M_{300}$}

Because of (a) limited S/N and significant sky emission line residuals in the WiFeS spectra, (b) the frequent presence of BAL features blueward of CIV, and (c) the desire to compile a homogeneous dataset for the LF that includes QSOs from the literature, we estimate the QSO absolute magnitude at restframe $145 \mathrm{~nm}, M_{145}$, utilising a composite spectrum of bright QSOs from $z=1-2$
(Selsing et al. 2016, S16, hereafter). In comparison to other QSO composite spectra that incorporate lower-luminosity objects (e.g., Telfer et al. 2002; Vanden Berk et al. 2001), the S16 composite ${ }^{7}$ provides a better match to the low-equivalent-width QSOs that one typically finds at high luminosity (as expected from the Baldwin Effect; Baldwin 1977; Rakshit, Stalin, \& Kotilainen 2020).

The $\mathrm{S} 16$ composite is convolved with the relevant filter curves to normalise the spectrum with the observed photometry. The $z-$ band filter curves for SMSS and SDSS, and the $H$-band filter curve for VISTA were retrieved from the Spanish Virtual Observatory (SVO) Filter Profile Service ${ }^{8}$ (Rodrigo, Solano, \& Bayo 2012; Rodrigo \& Solano 2020).

We validated the application of the S16 composite by comparing the associated $M_{145}$ predictions against the subsample of W16/Y16 data that was drawn from SDSS spectroscopy (in contrast to their own follow-up data, calibrated from SDSS $i$-band photometry). From 52 QSOs, the median $M_{145}$ difference, after adding $0.07 \mathrm{mag}$ to the values from Y16 ${ }^{9}$, was only 0.06 mag (with the S16 composite slightly brighter), with an RMS of just 0.12 mag. We take this as evidence that the combination of the observed photometry and the $\mathrm{S} 16$ composite spectrum will give robust results for computing the $M_{145}$ LFs. ${ }^{10}$

For $M_{300}$, we apply a similar procedure, but based on the $H$ band photometry (measured or inferred). From the S16 composite, $M_{145}-M_{300}=0.53 \mathrm{mag}$, but extrapolating the $M_{300}$ magnitudes from $z$-band would typically produce a slightly brighter $M_{300}$ value than our $H$-band-based estimates (up to $0.5 \mathrm{mag}$ ). This indicates that the spectral slopes of our QSOs are slightly bluer than the composite, as confirmed by the mean colour of the QSOs in our sample, $M_{145}-M_{300}=0.38$ mag. The bluer colours are consistent with the findings of Xie et al. (2016), in which more luminous QSOs had bluer UV spectral slopes. However, by anchoring our $M_{300}$ estimates to $H$-band, we minimise any associated error arising from variations in the spectral slope.

Cumulative number counts for $M_{145}$ and $M_{300}$ are shown in the right-hand panels of Fig. 5, split into our two redshift ranges.

\section{LUMINOSITY FUNCTIONS}

At the bright end, the number counts in the Southern sample exceed the number counts used previously for deriving the luminosity function of high-redshift QSOs. This allows us to be less dependent on completeness corrections. Hence, we re-derive the luminosity

7 Retrieved from https://github.com/jselsing/QuasarComposite 8 http://svo2.cab.inta-csic.es/theory/fps/

9 The Y16 cosmological parameters $\left(\Omega_{\Lambda}=0.728\right)$ predict 3.3 per cent larger luminosity distances at $z=5$ relative to ours.

10 In Paper I (and the initial arXiv version of this work), our $M_{145}$ estimates relied on simple interpolation between the SMSS $i_{\mathrm{psf}}$ and $z_{\mathrm{psf}}$ photometry. However, by ignoring the contributions of the UV emission lines, either photometric interpolation or the assumption of a fixed power-law continuum anchored to a single photometric band are likely to yield $M_{145}$ estimates that are systematically too bright. In particular, over the redshift interval $z=4.7-5.4$, the $\mathrm{S} 16$ composite gives $M_{145}$ values between 0.12 and 0.25 mag fainter than suggested by a $\left(\alpha_{v}=-0.3\right)$ power-law continuum anchored to $z$-band. Recent work by Grazian et al. (2021) at $z \sim 5$ have used similar power-law estimates (anchored to SMSS $i$-band), and our compositebased $M_{145}$ values are, in the mean, 0.18 mag fainter for the same 14 objects. Such use of $i$-band is particularly fraught as the redshift increases above $z=4.8$, where the flux through the filter is first boosted by Ly $\alpha$ emission, and then is soon diminished by Ly $\alpha$ absorption. 
function for the bright end and update estimates of the luminosity density and redshift evolution. Using the S16 composite spectrum, we can relate the apparent-magnitude completeness limit of $z_{\mathrm{PSF}}=18.7 \mathrm{ABmag}$ to a absolute-magnitude limit ranging from $M_{145}=-27.25 \mathrm{ABmag}$ at $z=4.4$ to $-27.73 \mathrm{ABmag}$ at $z=5.4$, and from $M_{300} \simeq-27.8 \mathrm{ABmag}$ at $z=4.4$ to $-28.3 \mathrm{ABmag}$ at $z=5.4$ (although we note that the $M_{300}$ magnitudes used in the LF calculation are estimated from $H$-band rather than extrapolating all the way from $z$-band). In the half-magnitude bin fainter than $z_{\mathrm{PSF}}=18.7 \mathrm{ABmag}$, the completeness of our sample collapses from $>80$ per cent to $\sim 10$ per cent, which will show in the figures we present for the binned luminosity function.

Prior to the application of any magnitude constraints, this final sample contains 171 QSOs in our search area of $14,486 \mathrm{deg}^{2}$, which is defined by $\delta<+2^{\circ},|b|>15^{\circ}$ and the exclusion zones detailed in Sect. 2. This sample excludes our two highest-redshift QSOs as well as a large number of QSOs identified at $z<4$.4. One of the literature QSOs does not have $z$-band magnitude from SMSS DR3, and we use the value from NSC DR2 instead ( $z=18.68 \mathrm{ABmag})$. The sample is assumed to be overall $\sim 95$ per cent complete at $z_{\mathrm{PSF}}<18 \mathrm{ABmag}$ and $>80$ per cent at $z_{\mathrm{PSF}}<18.7 \mathrm{ABmag}$, which we take into account for our statistical analysis.

For $4.4 \leq z<4.7$, we have 54 QSOs brighter than $M_{145}=$ -27.33 ABmag and which are used in the LF fitting below. That sample has a median redshift of 4.52 . For the $4.7 \leq z<5.4$ sample, which covers the same redshift interval as Y16 (a median redshift of 4.83 vs. 4.92 from Y16), we retain 28 QSOs brighter than $M_{145}=-27.73 \mathrm{ABmag}$.

For the first time, NIR photometry yields direct measurements of $M_{300}$ for a wide-area sample of QSOs at the bright end. This is due to the simultaneously deep and wide coverage of the VHS, which has no equivalent in the Northern hemisphere, where the samples for previous determinations of the bright end of the luminosity functions were found. Similar to our treatment of $M_{145}$, we apply cuts in $M_{300}$ to define the samples for fitting the LFs. The thresholds of $M_{300}<-27.86$ and $-28.26 \mathrm{ABmag}$ for the low and high redshift bins, respectively, are the $\mathrm{S} 16$ equivalents for $z_{\mathrm{PSF}}=18.7 \mathrm{AB}$ mag. As discussed in Sect. 4.4, because the spectral slopes of our QSOs are, on average, bluer than the S16 composite spectrum, the $M_{300}$ thresholds we adopt from the $z$-band limit are conservative. Nonetheless, we end up with similar numbers of QSOs in the $M_{300}$ LF fits for our two redshift ranges: 49 and 30.

\subsection{Parametric luminosity function fits}

We compare our data with the best-fit model of the LF from Y16, which is parametrised with a double power-law as

$\Phi(M, z)=\frac{\Phi^{*}(z)}{10^{0.4(\alpha+1)\left(M-M^{*}\right)}+10^{0.4(\beta+1)\left(M-M^{*}\right)}}$,

having a turnover in the density centred at luminosity $M^{*}=$ $-26.98 \pm 0.23$ ABmag, a faint-end slope of $\alpha=-2.03$ and a bright-end slope of $\beta=-3.58 \pm 0.24$. The normalisation, in units of number mag ${ }^{-1} \mathrm{Mpc}^{-3}$, is taken to evolve as $\log _{10} \Phi^{*}(z)=-8.82-0.47(z-6)$ (Fan et al. 2001), and we evaluate it at the median redshifts of our two bins. As noted in Sect. 4.4, we also add $0.07 \mathrm{mag}$ to the $M^{*}$ value from Y16 and adjust their comoving volume, as their cosmological parameters predict larger luminosity distances at $z=5$. The incompleteness of the Southern sample at the faint end is evident as a slope change in the right-hand panels of Fig. 5, particularly in the higher redshift bin.

To fit the LF, we adopt a Maximum Likelihood approach like that used by Y16 (see Marshall et al. 1983). We incorporate our full completeness model and consider two recent versions of the $z \sim 5$ faint-end LF: those of Niida et al. (2020) and Kim et al. (2020), hereafter $\mathrm{N} 20$ and K20, respectively. To probe such faint regimes, both surveys naturally covered much smaller areas, 340 and $85 \mathrm{deg}^{2}$, respectively, which yielded 72 and 32 QSOs across the $M_{145}$ range of -23 to $-27 \mathrm{ABmag}^{11}$. We take their respective faint-end slopes and normalisations, and fit our bright QSO sample (28 QSOs) with the double power-law of Eq. 5.1. For each of the two faint-end models, we allow $M^{*}$ to vary as part of the fit, but adjust $\Phi^{*}$ in a manner that maintains the existing fit to the faint-end data, given their respective $\alpha$ parameters. In this way, we fit $\beta$ and $M^{*}$, but also recompute $\Phi^{*}$.

First, we utilised the N20 faint-end parameters, namely $\alpha=$ -2.00 and a faint-end normalisation that remains consistent with their combination of $\log _{10} \Phi^{*}=-8.26 \mathrm{mag}^{-1} \mathrm{Mpc}^{-3}$ for $M^{*}=$ -27.15 ABmag. (N20 fixed their bright-end slope based on a fit to Y16 and McGreer et al. (2013), but utilised an independent selection of bright QSOs from SDSS DR7 in their MLE fit.) We adopted the redshift evolution of $\Phi^{*}$ from Fan et al. (2001) to scale the normalisation from their mean redshift of $z=4.75$ to our mean redshift of 4.91 . With our bright-end data, we then derive $\beta=-3.60 \pm 0.37$ and $M^{*}=-27.09 \pm 0.30 \mathrm{ABmag}$, which gives $\log _{10} \Phi^{*}=-8.32 \mathrm{mag}^{-1} \mathrm{Mpc}^{-3}$.

We then performed a similar fit with the K20 parameters, i.e., $\alpha=-1.11$ and $\log _{10} \Phi^{*}=-7.35 \mathrm{mag}^{-1} \mathrm{Mpc}^{-3}$ for $M^{*}=$ $-25.81 \mathrm{ABmag}$ at $\langle z\rangle=4$.95. (In contrast to N20, K20 explicitly used the Y16 data to constrain the bright end in their MLE fit.) We find $\beta=-3.38 \pm 0.32$ and $M^{*}=-25.87 \pm 0.33 \mathrm{ABmag}$, implying $\log _{10} \Phi^{*}=-7.33 \mathrm{mag}^{-1} \mathrm{Mpc}^{-3}$.

The results from fitting with each faint-end constraint are provided in Table 4, and the bright-end LFs are overplotted as straight lines in panels (B) and (F) of Fig. 5 (using the parameters from the K20 faint end). We also plot the Y16 LF fit in Fig. 5(F), where we have scaled their parameters to our cosmology and to our search area.

The overall $z \sim 5$ LF shape is illustrated in Figure 6, with the prior results shown in thin lines, and the new fits shown in thick lines for high redshift (solid) and low redshift (dashed). For the N20 parameters, there is some tension with the QSO densities found at the faint end of our highly complete survey region, where the close proximity between $M^{*}$ and our faint-end cutoff of $-27.73 \mathrm{ABmag}$ results in a steeper bright-end slope. In contrast, the shallower slope of K20 (in conjunction with its high normalisation) has no direct impact on the bright-end fit, and $M^{*}$ is more than 1 mag fainter, well below our sample's completeness limit.

While the LF in the low-redshift bin is generally higher at all magnitudes, the steeper bright-end slopes may suggest a change in behaviour at the brightest magnitudes (see Sect. 5.2).

Previous work, principally based on the Y16 sample, found broadly similar slopes, with data-driven 'best' free fits ranging from $\beta=-3.94$ by $\mathrm{N} 20$ and $\beta=-3.80$ by Y16 to $\beta=-3.50$ by K20. At a slightly lower redshift of $z \sim 3.9$, Boutsia et al. (2021) found a similar bright-end slope of $\beta=-4.0_{-0.4}^{+0.6}$. The Extremely Luminous QSO Survey (ELQS; Schindler et al. 2019b) found bright-end slopes for the redshift range of $2.8<z<4.5$ in the range from $\beta=-4$ to -4.5 depending on the details of the fitting boundaries.

11 As illustrated by Harikane et al. (2021), at the faint end of the N20 and K20 absolute magnitude ranges, the population of UV sources is rapidly transitioning from being QSO-dominated to being galaxy-dominated, so contamination in the QSO samples becomes a serious concern. 


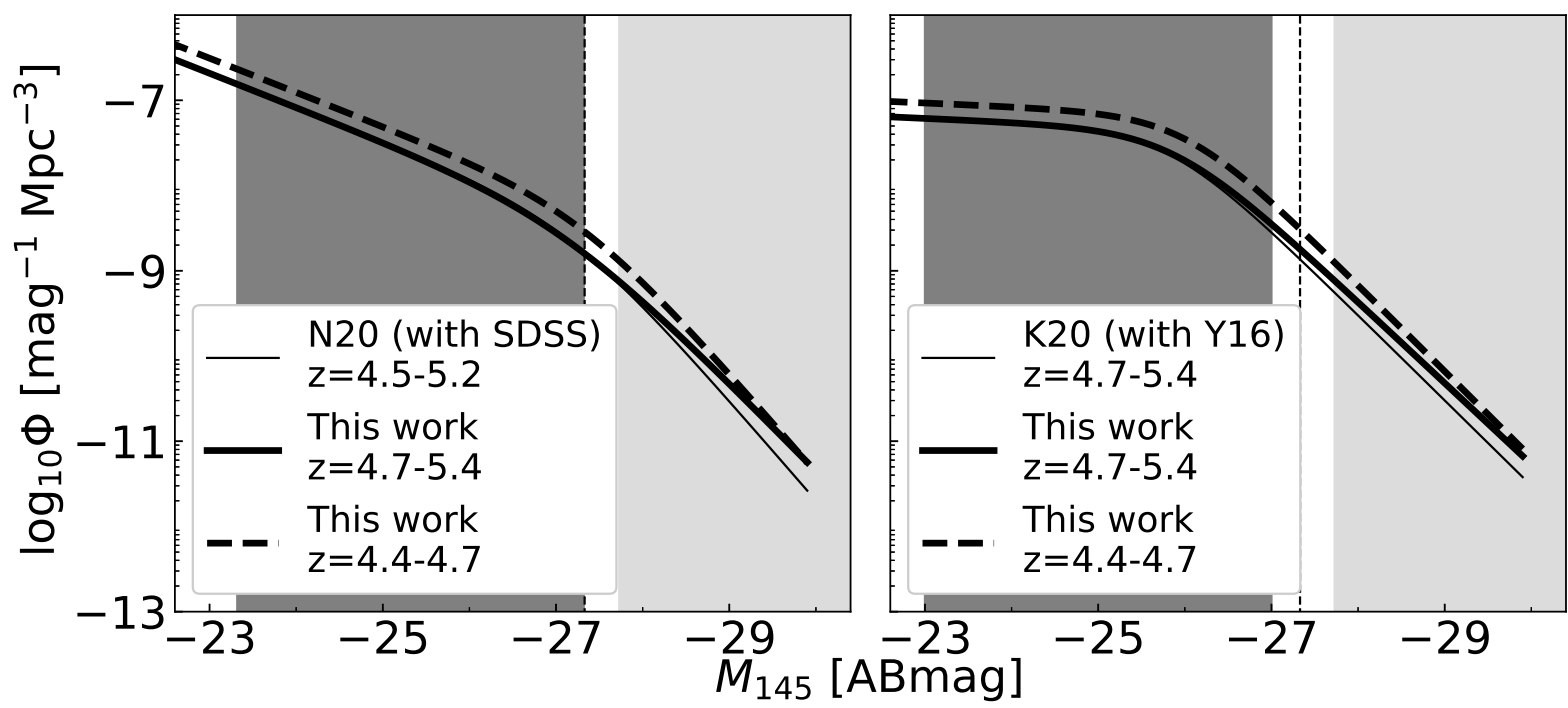

Figure 6. Parametrised QSO LF models at $z \sim 5$, combining our bright-end data with the faint-end slopes and normalisations of (left) N20 and (right) K20. The solid lines show the fits at $z=4.7-5.4$ : ours (thick), and the original N20/K20 models (thin). The bright-end fitting regions for our data are shown in the light grey shaded areas for $z=4.7-5.4$. Our fits to the $z=4.4-4.7$ sample are shown with thick dashed lines and the lower magnitude bounds for $z=4.4-4.7$ are shown as vertical dotted lines. The fits include sources up to a maximum of $M_{145}=-29.1$ ABmag. The faint-end samples cover the dark grey regions of $M_{145} \sim-23$ to $\sim-27 \mathrm{ABmag}$.

Table 4. Restframe $145 \mathrm{~nm}$ LF parameters for double power-law.

\begin{tabular}{ccccc}
\hline median z & $\beta$ & $M^{*}$ & $\alpha$ & $\log _{10} \Phi^{*}$ \\
\hline \multicolumn{5}{c}{ Faint-end constraints from Kim et al. (2020) } \\
\hline 4.52 & $-3.53 \pm 0.24$ & $-26.02 \pm 0.18$ & -1.11 & -7.17 \\
4.83 & $-3.38 \pm 0.32$ & $-25.87 \pm 0.33$ & -1.11 & -7.33
\end{tabular}

Faint-end constraints from Niida et al. (2020)

\begin{tabular}{lllll}
\hline 4.52 & $-3.92 \pm 0.32$ & $-27.32 \pm 0.13$ & -2.00 & -8.24 \\
4.83 & $-3.60 \pm 0.37$ & $-27.09 \pm 0.30$ & -2.00 & -8.32 \\
\hline
\end{tabular}

For the $M_{300} \mathrm{LF}$ at $z \sim 5$, we lack the deep $H$-band photometric data to provide the counterparts to the N20 and K20 constraints, so we focus on fitting just a single power-law to the bright end of the LF. Applying the same MLE technique to that above, we fit the LF as a pure power-law. Retaining the redshift dependence of Fan et al. (2001) for the LF normalisation, we fit the $\beta$ and $\Phi$ values at $M_{145}=$ -28.5 ABmag at $z=4.4-4.7$, and $M_{145}=-28.75$ ABmag at $z=4.7-5.4$.

At $z=4.4-4.7$, from 49 QSOs, we find the best fit to the bright end of the $M_{300} \mathrm{LF}$ to be $\beta=-3.18 \pm 0.22$ and $\log _{10} \Phi\left(M_{300}=-28.5\right)=-9.18 \pm 0.05 \mathrm{mag}^{-1} \mathrm{Mpc}^{-3}$. In the $z=4.7-5.4$ bin, from 30 QSOs, we find $\beta=-3.43 \pm 0.32$ and $\log _{10} \Phi\left(M_{300}=-28.75\right)=-9.56 \pm 0.06 \mathrm{mag}^{-1} \mathrm{Mpc}^{-3}$. The LF fits are shown with straight lines in panels (D) and (H) of Fig. 5.

\subsection{Redshift evolution and downsizing}

Decades of survey work in the evolution of galaxies and QSOs have established a paradigm of cosmic downsizing: in this paradigm, the galaxies reaching the highest stellar masses have formed the bulk of their stars at high redshift during short epochs of particularly intense star formation; in contrast, galaxies of progressively lower mass formed at later cosmic epochs during more extended and less intense activity (e.g. Cowie et al. 1996, among many others). Given well-established relations between the mass of central supermassive black holes and the mass of their host galaxy bulges (Ferrarese \& Merritt 2000; Kormendy \& Ho 2013), we expect that the most massive black holes have thus grown most rapidly in the most luminous QSOs at high redshift. Surveys of QSOs have revealed a similar and matching trend of higher-luminosity objects peaking in activity at higher redshift, while lower-luminosity QSOs seem to have later activity peaks (e.g. Hasinger, Miyaji, \& Schmidt 2005; Hopkins, Richards, \& Hernquist 2007).

However, most of the past works could not draw on complete samples of QSOs at the extreme luminosities of $M_{145}<-28$, and hence we now compare the newest bright-end measurements of the QSO LF spanning the redshift range from $z \sim 4$ to $z \sim 6$ to reassess its evolution. In Fig. 7, we complement our work and that of W16/Y16 with the LFs from ELQS and from Boutsia et al. (2021) on the lower-z side and with the LF by Jiang et al. (2016) on the higher-z end. Here, we visualise space densities from the actual best-fit parametrisations, because, due to the range of different bright-end slopes, it would be insufficient to consider only $M^{*}$ and $\Phi^{*}$ parameters.

The evolution parameter of $\gamma=d \log \Phi(M) / d z=0.47$ estimated by Fan et al. (2001) is shown in Fig. 7 as well and broadly consistent with the evolution at the highest redshifts. We see no strong evidence of a peak or plateau, although there is a hint of flattening around redshift 3 to 4 .

\section{SUMMARY}

We continued our program to search for QSOs at $z \geq 4$ in the Southern hemisphere using data from SkyMapper, Gaia, and several infrared surveys. We focus on the ultra-luminous end of the QSO 


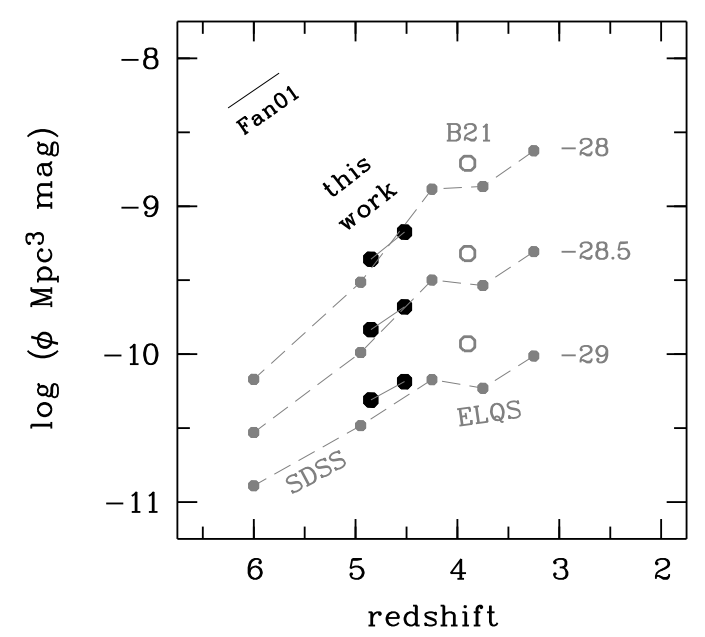

Figure 7. Evolution of $\Phi(M)$ with redshift at three $M_{145}$ points. Large symbols are from Southern samples in the SkyMapper survey area, with black points from this work and the grey open symbol from Boutsia et al. (2021). Small points are dominated by Northern samples including Jiang et al. (2016) at $z \simeq 6$, Y16 at $z \simeq 5$ and ELQS points at $z<4.5$. The Fan et al. (2001) evolution of $d \log \Phi^{*} / d z$ is shown in the top left corner.

distribution, the luminosity range of $M_{145}<-27 \mathrm{ABmag}$, where true QSOs are outnumbered by cool stars to the most extreme degree possible. In this paper, we extend the search area to an effective $14486 \mathrm{deg}^{2}$. We have obtained full spectroscopic coverage of our candidate list at $z_{\mathrm{PSF}}<18.7 \mathrm{ABmag}$, at which depth we are $>80$ per cent complete.

We discover 126 new QSOs with $M_{145}<-27$ ABmag, on top of 21 such objects presented by Paper I, as well as a small number of less luminous objects. Previously, the most complete sample of bright high-redshift QSOs was that of W16 \& Y16, found within a similarly sized search area in the Northern sky, with a focus on the redshift range of $4.7<z<5.4$.

We use our sample to redetermine the bright end of the QSO luminosity function at restframe $145 \mathrm{~nm}$, updating that of Y16, and also present for the first time the bright end of the high-redshift QSO luminosity function at restframe $300 \mathrm{~nm}$, capitalising on the depth of wide-field NIR photometry available in the Southern hemisphere.

We measure the slope of the $M_{145}$ luminosity at $z=4.7-5.4$ as $\beta=-3.38 \pm 0.32$ and $-3.60 \pm 0.37$, depending on the faint-end constraint that is used, both of which are in line with several earlier estimates. In addition, we measure $\beta=-3.43 \pm 0.32$ for $M_{300}$ from a fit with no faint-end constraint.

In the $z=4.4-4.7$ range, we measure slightly steeper brightend slopes of $\beta=-3.53 \pm 0.24$ and $-3.92 \pm 0.32$ for the $M_{145} \mathrm{LF}$, and $\beta=-3.18 \pm 0.22$ for $M_{300}$.

A final determination of the QSO space densities at the luminous end and their evolution will require completing searches in the Northern hemisphere to create a complete all-sky sample of high-redshift QSOs. While this is one of our next steps, another is to push the completeness limit deeper to $z_{\mathrm{PSF}} \approx 19 \mathrm{ABmag}$ in order to obtain more robust statistics in the region where this sample connects to and overlaps with deeper literature samples. This, however, will require significant observational effort given that the contamination by stars increases with the fading discriminating power of Gaia parallaxes and proper motions at $19 \mathrm{ABmag}$ and beyond.

\section{ACKNOWLEDGEMENTS}

A number of observers contributed spectroscopy of candidates through time-swapping arrangements or extreme generosity, and we thank them for their efforts: Harrison Abbot, Katie Auchettl, and Mike Bessell. We also thank the referee for suggestions that greatly improved the quality of the manuscript. CAO was supported by the Australian Research Council (ARC) through Discovery Project DP190100252. The national facility capability for SkyMapper has been funded through ARC LIEF grant LE130100104 from the Australian Research Council, awarded to the University of Sydney, the Australian National University, Swinburne University of Technology, the University of Queensland, the University of Western Australia, the University of Melbourne, Curtin University of Technology, Monash University and the Australian Astronomical Observatory. SkyMapper is owned and operated by The Australian National University's Research School of Astronomy and Astrophysics. The survey data were processed and provided by the SkyMapper Team at ANU. The SkyMapper node of the All-Sky Virtual Observatory (ASVO) is hosted at the National Computational Infrastructure (NCI). Development and support the SkyMapper node of the ASVO has been funded in part by Astronomy Australia Limited (AAL) and the Australian Government through the Commonwealth's Education Investment Fund (EIF) and National Collaborative Research Infrastructure Strategy (NCRIS), particularly the National eResearch Collaboration Tools and Resources (NeCTAR) and the Australian National Data Service Projects (ANDS). This project has made use of data from the European Space Agency (ESA) mission Gaia (https://www.cosmos.esa.int/gaia), processed by the Gaia Data Processing and Analysis Consortium (DPAC, https://www . cosmos.esa.int/web/gaia/dpac/consortium). Funding for the DPAC has been provided by national institutions, in particular the institutions participating in the Gaia Multilateral Agreement. This publication makes use of data products from the Wide-field Infrared Survey Explorer, which is a joint project of the University of California, Los Angeles, and the Jet Propulsion Laboratory/California Institute of Technology, and NEOWISE, which is a project of the Jet Propulsion Laboratory/California Institute of Technology. WISE and NEOWISE are funded by the National Aeronautics and Space Administration. We have used data products from the Two Micron All Sky Survey, which is a joint project of the University of Massachusetts and the Infrared Processing and Analysis Center/California Institute of Technology, funded by the National Aeronautics and Space Administration and the National Science Foundation. This paper uses data from the VISTA Hemisphere Survey ESO programme ID: 179.A-2010 (PI. McMahon). Based on observations obtained as part of the VISTA Hemisphere Survey, ESO Progam, 179.A-2010 (PI: McMahon). The VISTA Data Flow System pipeline processing and science archive are described in Irwin et al. (2004), Hambly et al. (2008) and Cross et al. (2012). This publication has made use of data from the VIKING survey from VISTA at the ESO Paranal Observatory, programme ID 179.A-2004. Data processing has been contributed by the VISTA Data Flow System at CASU, Cambridge and WFAU, Edinburgh. This research has made use of the SVO Filter Profile Service (http://svo2.cab.inta-csic.es/theory/fps/) supported from the Spanish MINECO through grant AYA201784089. The results presented here have utilised modules from the ScIPy software package (Jones et al. 2001). 


\section{DATA AVAILABILITY}

The SMSS data underlying this article are available at the SkyMapper node of the All-Sky Virtual Observatory (ASVO), hosted at the National Computational Infrastructure (NCI) at https://skymapper.anu.edu.au. The data from SMSS Data Release 3 are currently accessible only to Australia-based researchers and their collaborators.

\section{REFERENCES}

Baldwin J. A., 1977, ApJ, 214, 679. doi:10.1086/155294

Bessell M., Bloxham G., Schmidt B., Keller S., Tisserand P., Francis P., 2011, PASP, 123, 789. doi:10.1086/660849

Boutsia K., Grazian A., Fontanot F., Giallongo E., Menci N., Calderone G., Cristiani S., et al., 2021, ApJ, 912, 111. doi:10.3847/1538-4357/abedb5

Calderone G., Boutsia K., Cristiani S., Grazian A., Amorin R., D’Odorico V., Cupani G., et al., 2019, ApJ, 887, 268. doi:10.3847/1538-4357/ab510a

Casagrande L., VandenBerg D. A., 2018, MNRAS, 479, L102

Chambers K. C., Magnier E. A., Metcalfe N., Flewelling H. A., Huber M. E., Waters C. Z., Denneau L., et al., 2016, arXiv, arXiv:1612.05560

Childress, M. J., Vogt, F. P. A., Nielsen, J., \& Sharp, R. G. 2014, Ap\&SS, 349,617

Cowie L. L., Songaila A., Hu E. M., Cohen J. G., 1996, AJ, 112, 839. doi: $10.1086 / 118058$

Cross N. J. G., Collins R. S., Mann R. G., Read M. A., Sutorius E. T. W., Blake R. P., Holliman M., et al., 2012, A\&A, 548, A119. doi:10.1051/0004-6361/201219505

Dopita, M., Rhee, J., Farage, C. et al. 2010, Ap\&SS, 327, 245

Edge A., Sutherland W., Kuijken K., Driver S., McMahon R., Eales S., Emerson J. P., 2013, ESO Messenger, 154, 32

Fan X., Strauss M. A., Schneider D. P., Gunn J. E., Lupton R. H., Becker R. H., Davis M., et al., 2001, AJ, 121, 54. doi:10.1086/318033

Ferrarese L., Merritt D., 2000, ApJL, 539, L9. doi:10.1086/312838

Flesch E. W., 2015, PASA, 32, e010

Gaia Collaboration, Brown A. G. A., Vallenari A., Prusti T., de Bruijne J. H. J., Babusiaux C., Bailer-Jones C. A. L., et al., 2018, A\&A, 616, A1. doi:10.1051/0004-6361/201833051

Gaia Collaboration, Brown A. G. A., Vallenari A., Prusti T., de Bruijne J. H. J., Babusiaux C., Biermann M., et al., 2021, A\&A, 649, A1. doi:10.1051/0004-6361/202039657

Grazian A., Giallongo E., Boutsia K., Calderone G., Cristiani S., Cupani G., Fontanot F., et al., 2021, ApJ (in press, arXiv:2110.13736)

Guarneri F., Calderone G., Cristiani S., Fontanot F., Boutsia K., Cupani G., Grazian A., et al., 2021, MNRAS, 506, 2471. doi:10.1093/mnras/stab1867

Hambly N. C., Collins R. S., Cross N. J. G., Mann R. G., Read M. A., Sutorius E. T. W., Bond I., et al., 2008, MNRAS, 384, 637. doi:10.1111/j.13652966.2007.12700.x

Harikane Y., Ono Y., Ouchi M., Liu C., Sawicki M., Shibuya T., Behroozi P. S., et al., 2021, ApJS (in press, arXiv:2108.01090)

Hasinger G., Miyaji T., Schmidt M., 2005, A\&A, 441, 417. doi:10.1051/0004-6361:20042134

Hinton S. R., Davis T. M., Lidman C., Glazebrook K., Lewis G. F., 2016, Astronomy \& Computing, 15, 61

Hopkins P. F., Richards G. T., Hernquist L., 2007, ApJ, 654, 731. doi:10.1086/509629

Irwin M. J., Lewis J., Hodgkin S., Bunclark P., Evans D., McMahon R., Emerson J. P., et al., 2004, SPIE, 5493, 411. doi:10.1117/12.551449

Jiang L. et al., 2016, ApJ, 833, 222

Jones E. et al., 2001, SciPy: Open source scientific tools for Python, http://www.scipy.org/

Karachentsev I. D., Makarov D. I., Kaisina E. I., 2013, AJ, 145, 101. doi:10.1088/0004-6256/145/4/101

Kim Y., Im M., Jeon Y., Kim M., Pak S., Hyun M., Taak Y. C., et al., 2020, ApJ, 904, 111. doi:10.3847/1538-4357/abc0ea (K20)
Kormendy J., Ho L. C., 2013, ARA\&A, 51, 511. doi:10.1146/annurev-astro082708-101811

Lyke B. W., Higley A. N., McLane J. N., Schurhammer D. P., Myers A. D., Ross A. J., Dawson K., et al., 2020, ApJS, 250, 8. doi:10.3847/15384365/aba623

Marocco F., Eisenhardt P. R. M., Fowler J. W., Kirkpatrick J. D., Meisner A. M., Schlafly E. F., Stanford S. A., et al., 2021, ApJS, 253, 8. doi: $10.3847 / 1538-4365 /$ abd805

Marshall H. L., Tananbaum H., Avni Y., Zamorani G., 1983, ApJ, 269, 35. doi:10.1086/161016

McGreer I. D., Jiang L., Fan X., Richards G. T., Strauss M. A., Ross N. P., White M., et al., 2013, ApJ, 768, 105. doi:10.1088/0004$637 \mathrm{X} / 768 / 2 / 105$

McGreer I. D., Fan X., Jiang L., Cai Z., 2018, AJ, 155, 131. doi:10.3847/1538-3881/aaaab4

McMahon, R. G., Banerji, M., Gonzalez, E. et al. 2013, The Messenger, 154,35

Mortlock D. J., Patel M., Warren S. J., Hewett P. C., Venemans B. P., McMahon R. G., Simpson C., 2012, MNRAS, 419, 390. doi:10.1111/j.13652966.2011.19710.x

Nidever D. L., Dey A., Fasbender K., Juneau S., Meisner A. M., Wishart J., Scott A., et al., 2021, AJ, 161, 192. doi:10.3847/1538-3881/abd6e1

Niida M., Nagao T., Ikeda H., Akiyama M., Matsuoka Y., He W., Matsuoka K., et al., 2020, ApJ, 904, 89. doi:10.3847/1538-4357/abbe11 (N20)

Onken, C. A., Wolf, C., Shao, L., Luvaul, L. C. et al. 2019, PASA, 36, 33

Onken C. A., Bian F., Fan X., Wang F., Wolf C., Yang J., 2020, MNRAS, 496, 2309. doi:10.1093/mnras/staa 1635

Rakshit S., Stalin C. S., Kotilainen J., 2020, ApJS, 249, 17. doi: $10.3847 / 1538-4365 / a b 99 \mathrm{c} 5$

Reed S. L., McMahon R. G., Martini P., Banerji M., Auger M., Hewett P. C., Koposov S. E., et al., 2017, MNRAS, 468, 4702. doi:10.1093/mnras/stx728

Richards G. T., Strauss M. A., Fan X., Hall P. B., Jester S., Schneider D. P., Vanden Berk D. E., et al., 2006, AJ, 131, 2766. doi:10.1086/503559

Richards G. T., Deo R. P., Lacy M., Myers A. D., Nichol R. C., Zakamska N. L., Brunner R. J., et al., 2009, AJ, 137, 3884. doi:10.1088/0004$6256 / 137 / 4 / 3884$

Riello M., De Angeli F., Evans D. W., Montegriffo P., Carrasco J. M., Busso G., Palaversa L., et al., 2021, A\&A, 649, A3. doi:10.1051/0004$6361 / 202039587$

Rodrigo C., Solano E., Bayo A., 2012, ivoa.rept. doi:10.5479/ADS/bib/2012ivoa.rept.1015R

Rodrigo C., Solano E., 2020, sea..conf, 182

Ryan-Weber E. V., Pettini M., Madau P., Zych B. J., 2009, MNRAS, 395, 1476

Schindler J.-T., Fan X., Huang Y.-H., Yue M., Yang J., Hall P. B., Wenzl L., et al., 2019a, ApJS, 243, 5. doi:10.3847/1538-4365/ab20d0

Schindler J.-T., Fan X., McGreer I. D., Yang J., Wang F., Green R., Fynbo J. P. U., et al., 2019b, ApJ, 871, 258. doi:10.3847/1538-4357/aaf86c

Schlafly E. F., Finkbeiner D. P., 2011, ApJ, 737, 103. doi:10.1088/0004637X/737/2/103

Schlegel D. J., Finkbeiner D. P., Davis M., 1998, ApJ, 500, 525

Selsing J., Fynbo J. P. U., Christensen L., Krogager J.-K., 2016, A\&A, 585, A87. doi:10.1051/0004-6361/201527096 (S16)

Simcoe, R. A., Cooksey, K. L., Matejek, M. et al. 2011, ApJ, 743, 21

Skrutskie, M. F., Cutri, R. M., Stiening, R., Weinberg, M. D., et al. 2006, AJ, 131, 1163

Telfer R. C., Zheng W., Kriss G. A., Davidsen A. F., 2002, ApJ, 565, 773. doi: $10.1086 / 324689$

Vanden Berk D. E., Richards G. T., Bauer A., Strauss M. A., Schneider D. P., Heckman T. M., York D. G., et al., 2001, AJ, 122, 549. doi: $10.1086 / 321167$

Wang, F., Wu, X.-B., Fan, X. et al. 2016, ApJ, 819, 24 (W16)

Wang S., Chen X., 2019, ApJ, 877, 116. doi:10.3847/1538-4357/ab1c61

Wenzl L., Schindler J.-T., Fan X., Andika I. T., Bañados E., Decarli R., Jahnke K., et al., 2021, AJ, 162, 72. doi:10.3847/1538-3881/ac0254 (W21)

Wolf C., Meisenheimer K., Röser H.-J., 2001, A\&A, 365, 660. 
doi:10.1051/0004-6361:20000474

Wolf, C., Onken, C. A., Luvaul, L. C. et al. 2018a, PASA, 35, 10. doi: 10.4225/41/593620ad5b574

Wolf, C., Bian, F., Onken, C. A., Schmidt, B. P., Tisserand, P. et al. 2018b, PASA, 35, 24

Wolf C., Hon W. J., Bian F., Onken C. A., Alonzi N., Bessell M. A., Li Z., et al., 2020, MNRAS, 491, 1970. doi:10.1093/mnras/stz2955 (Paper I)

Wright, E. L., Eisenhardt, P. R. M., Mainzer, A. K. et al. 2010, AJ, 140, 1868

Xie X., Shao Z., Shen S., Liu H., Li L., 2016, ApJ, 824, 38. doi:10.3847/0004-637X/824/1/38

Yang J. et al., 2016, ApJ, 829, 33 (Y16)

Yang J. et al., 2017, AJ, 153, 184

Yang J. et al., 2019, ApJ, 871, 199

York, D. G., Adelman, J., Anderson, J. E., Jr. et al. 2000, AJ, 120, 1579

\section{APPENDIX A: SPECTRUM GALLERY}

In the Figures below, we present the WiFeS spectra for the newly discovered QSOs from the redshift range $z=3.8-5.5$, ordered by RA. 

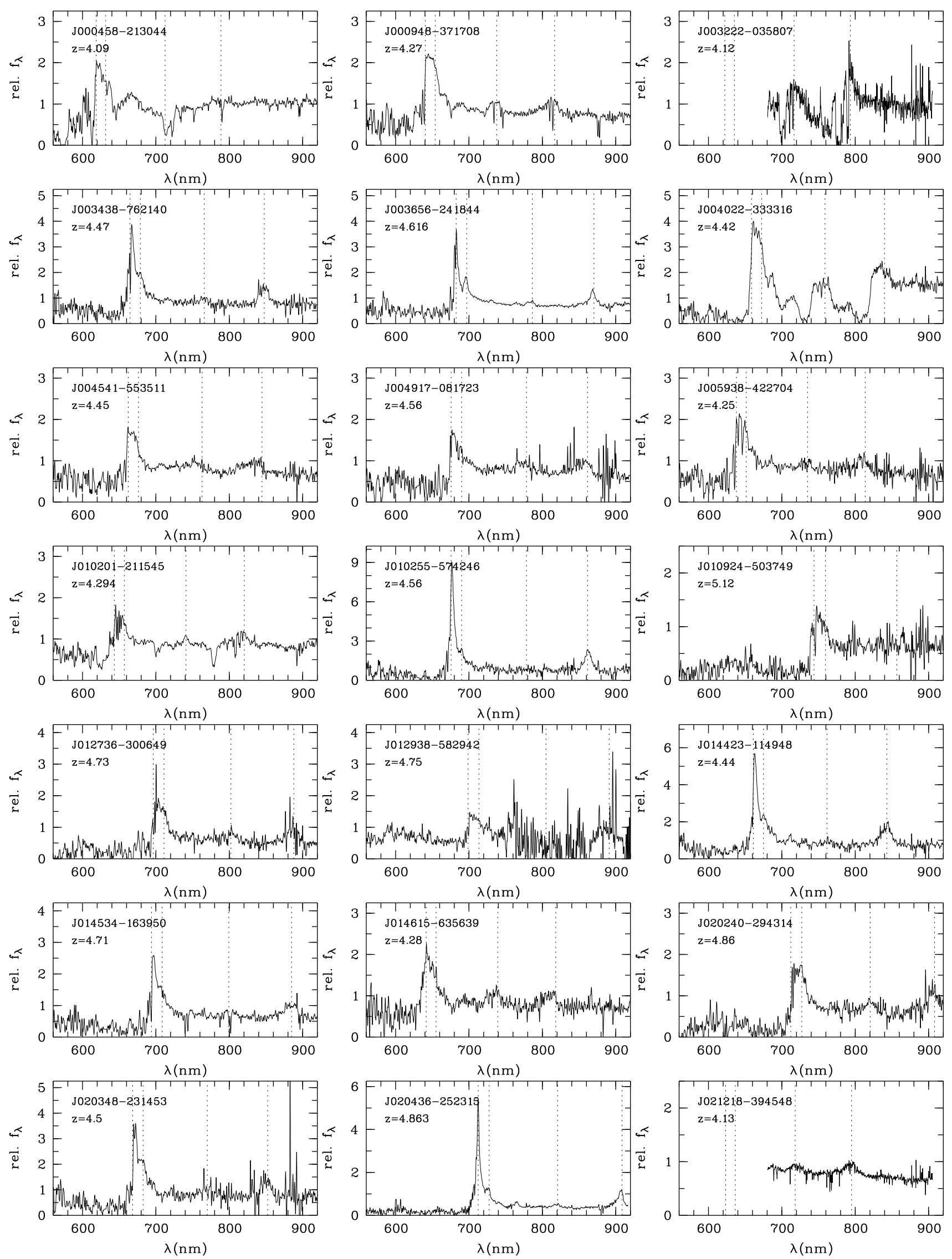

Figure A.1. Gallery of $3.8>z>5.5$ QSO spectra obtained in this work, ordered by RA, page 1. Vertical dashed lines indicate the wavelengths of Ly $\alpha$, Nv, $\mathrm{Si}$ IV, and $\mathrm{C}$ IV at our adopted redshift. 

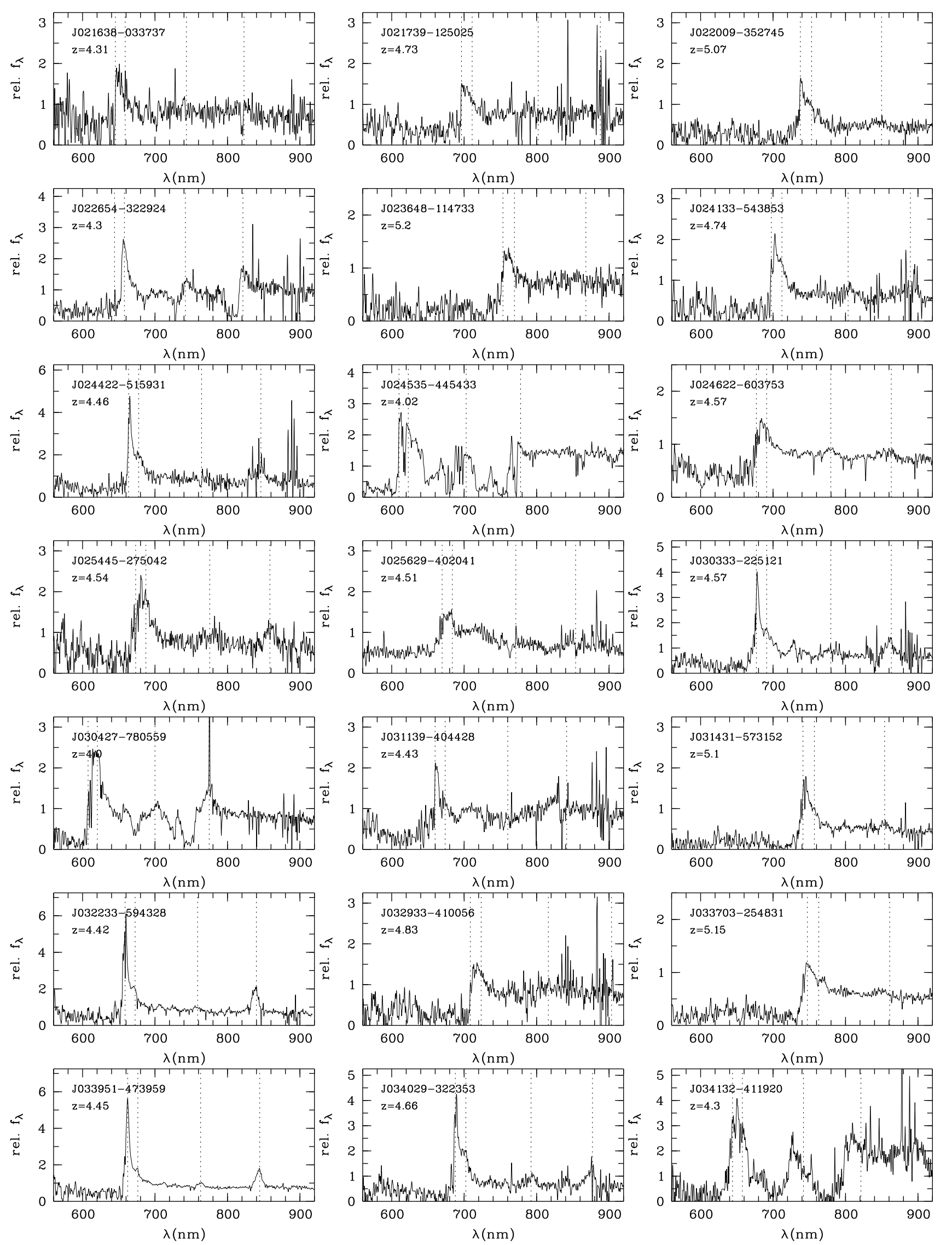

Figure A.2. Gallery of $3.8>z>5.5$ QSO spectra obtained in this work, ordered by RA, page 2 . 

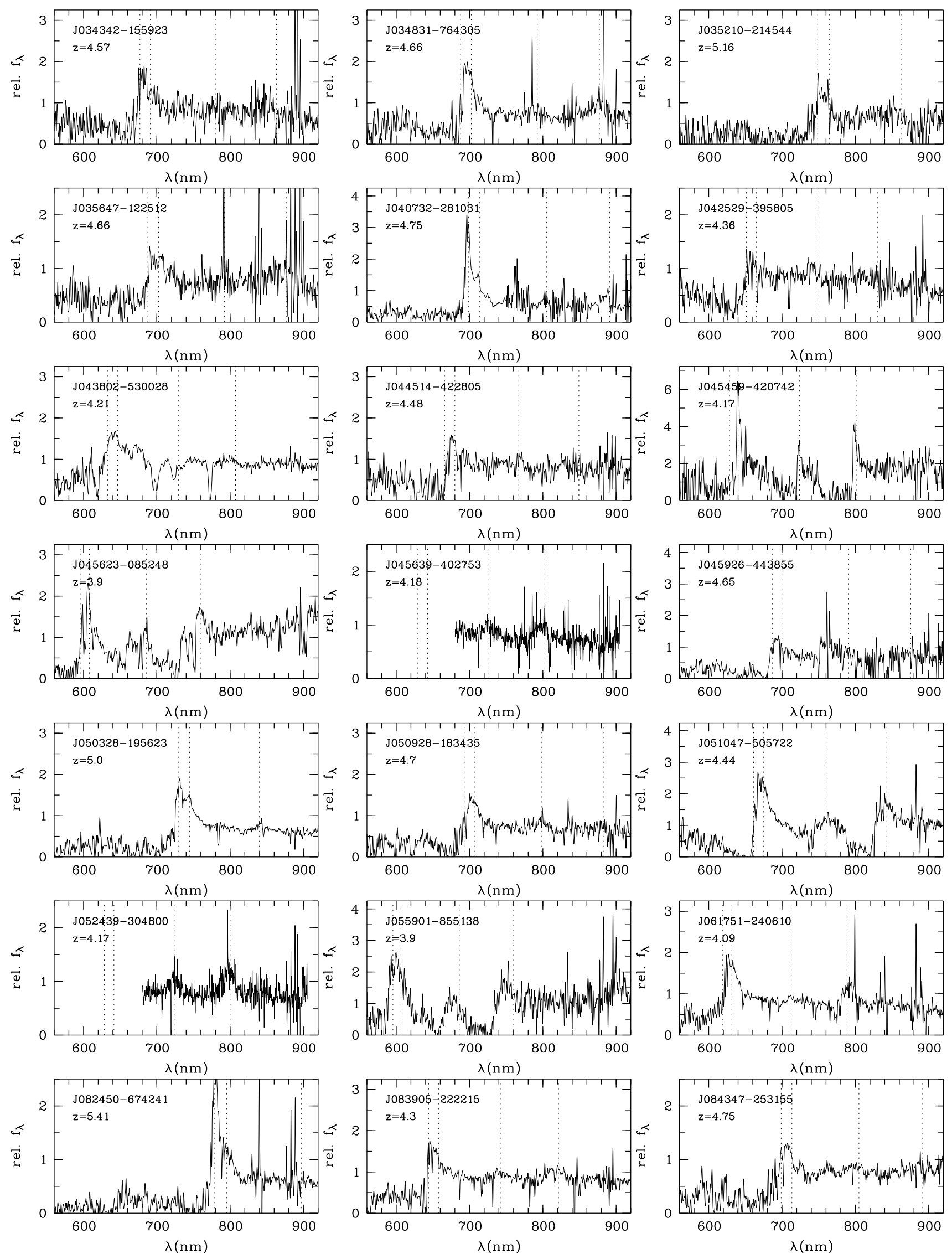

Figure A.3. Gallery of $3.8>z>5.5$ QSO spectra obtained in this work, ordered by RA, page 3 . 

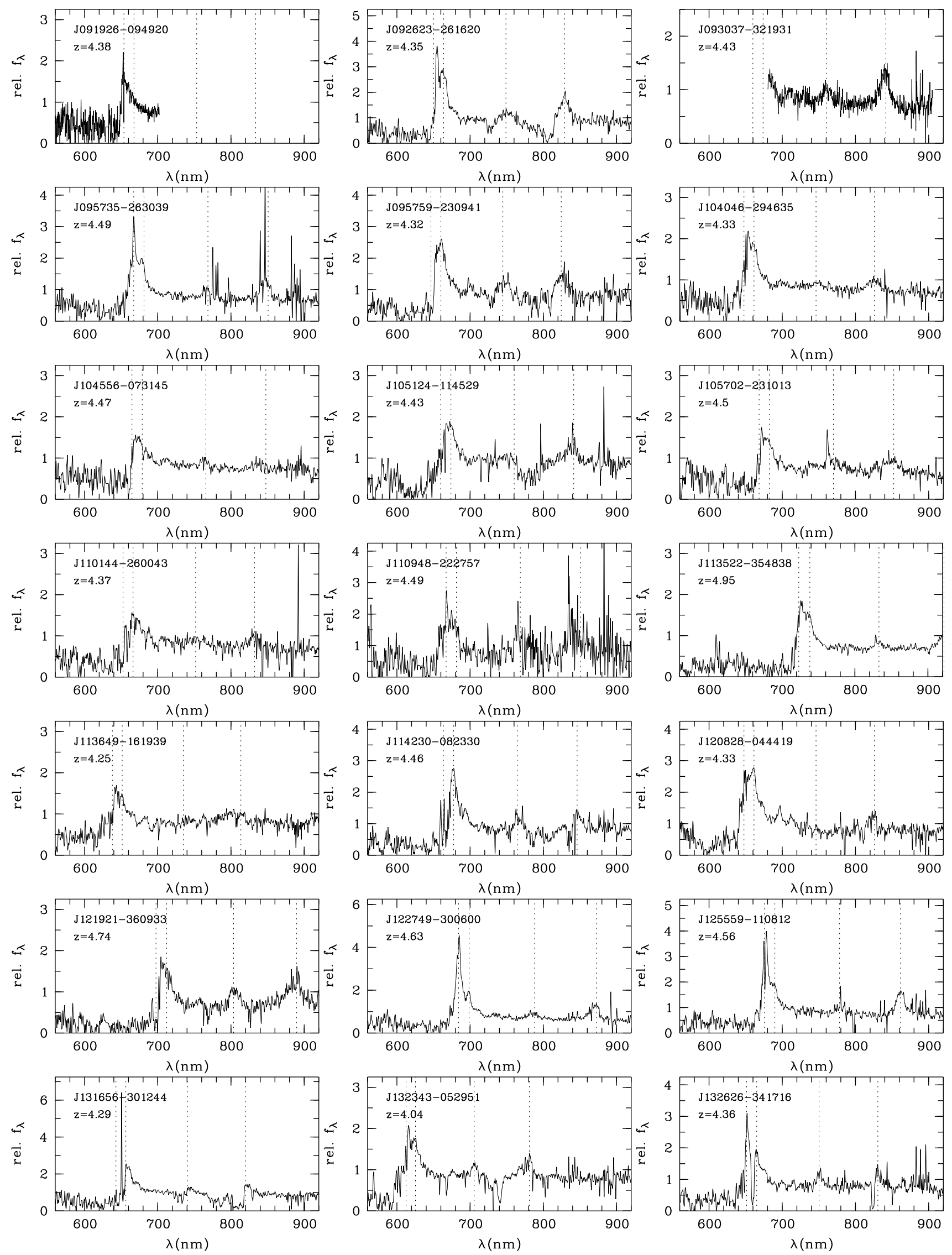

Figure A.4. Gallery of $3.8>z>5.5$ QSO spectra obtained in this work, ordered by RA, page 4 . 

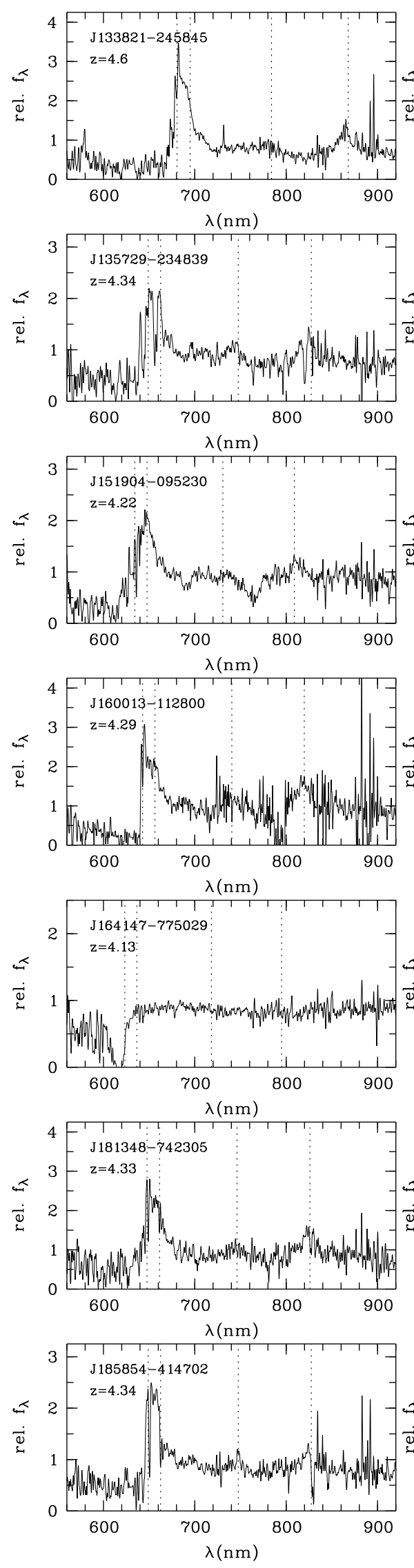
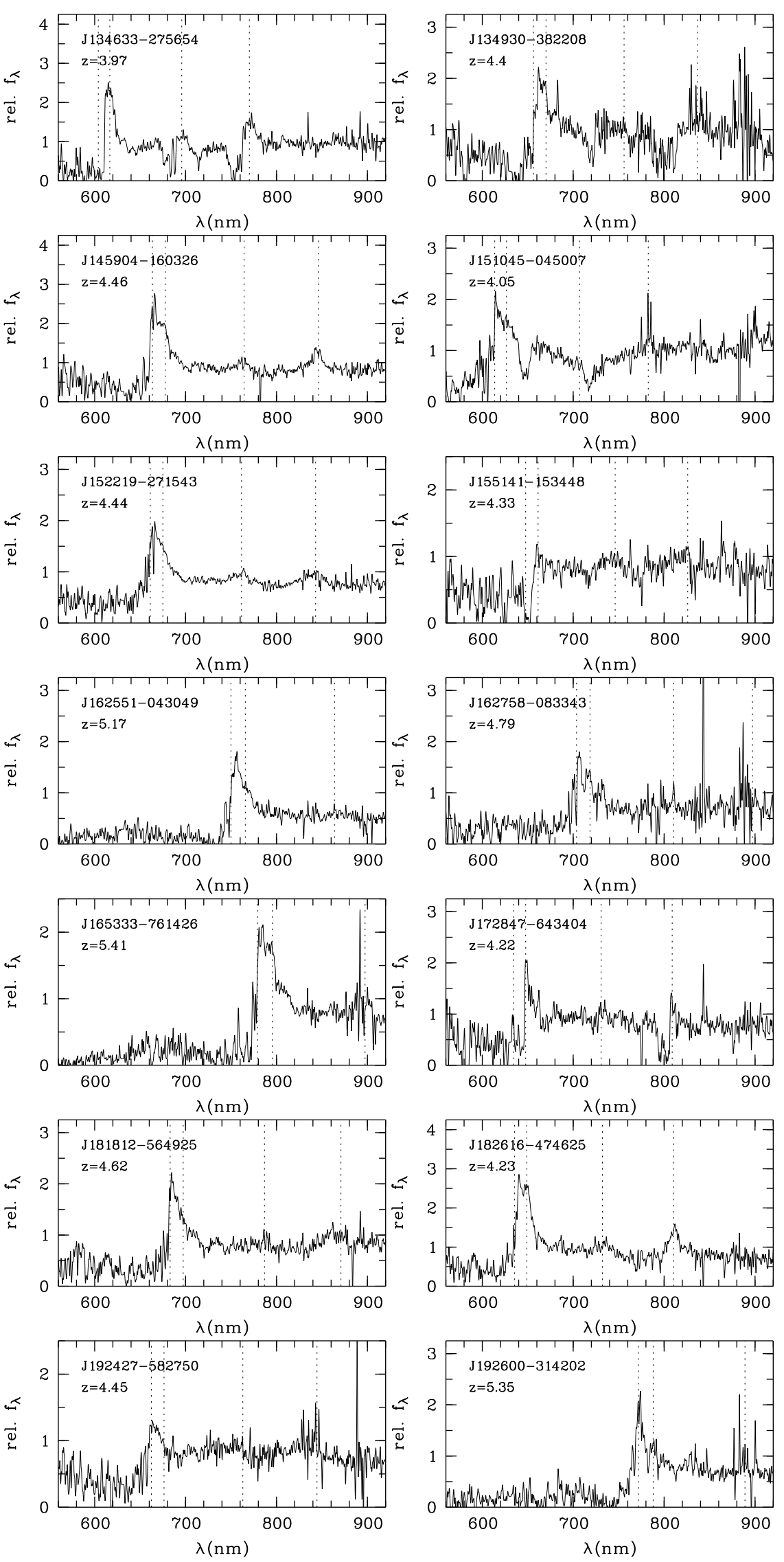

Figure A.5. Gallery of $3.8>z>5.5$ QSO spectra obtained in this work, ordered by RA, page 5 . 

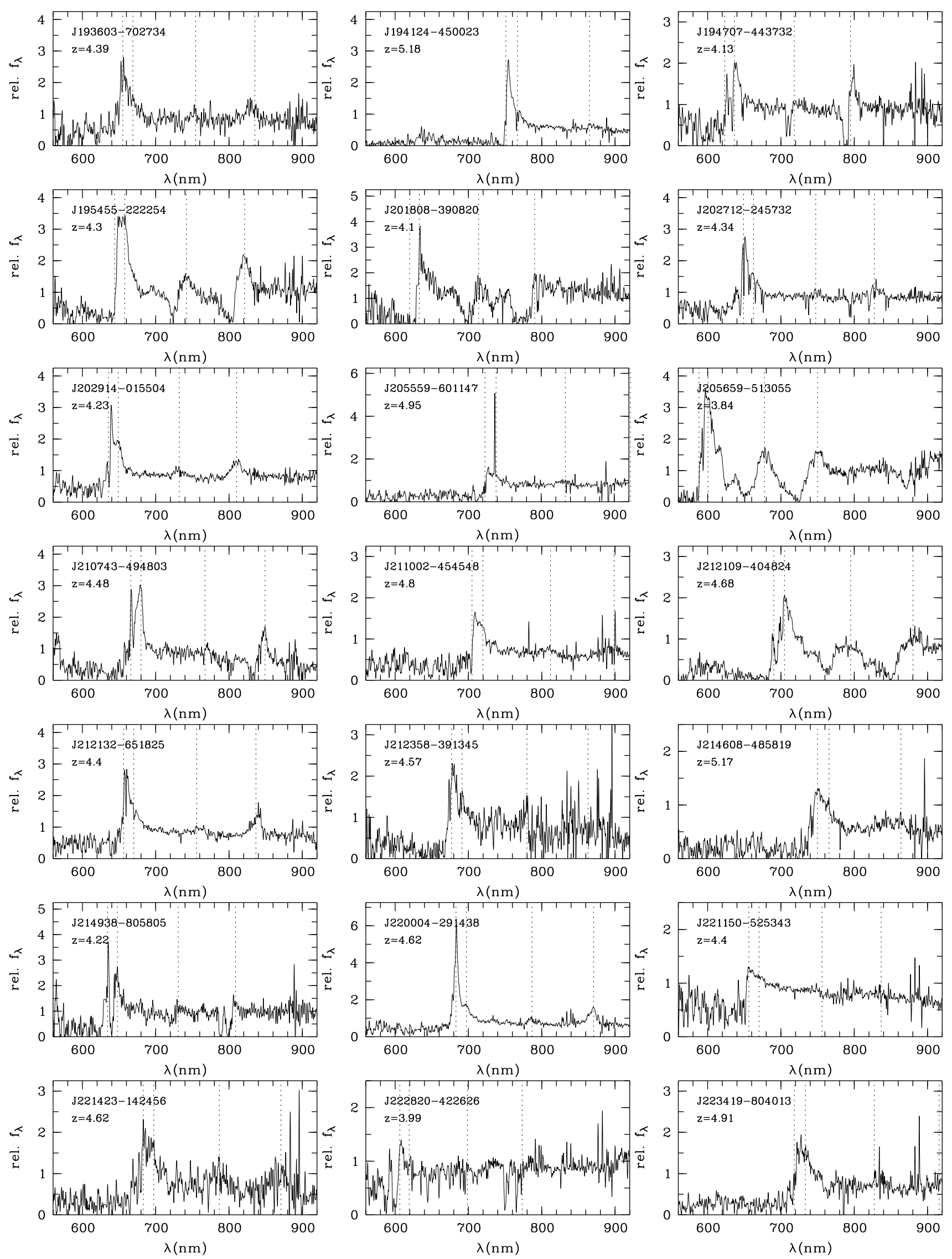

Figure A.6. Gallery of $3.8>z>5.5$ QSO spectra obtained in this work, ordered by RA, page 6 . 
Ultra-luminous quasars from SkyMapper. II.
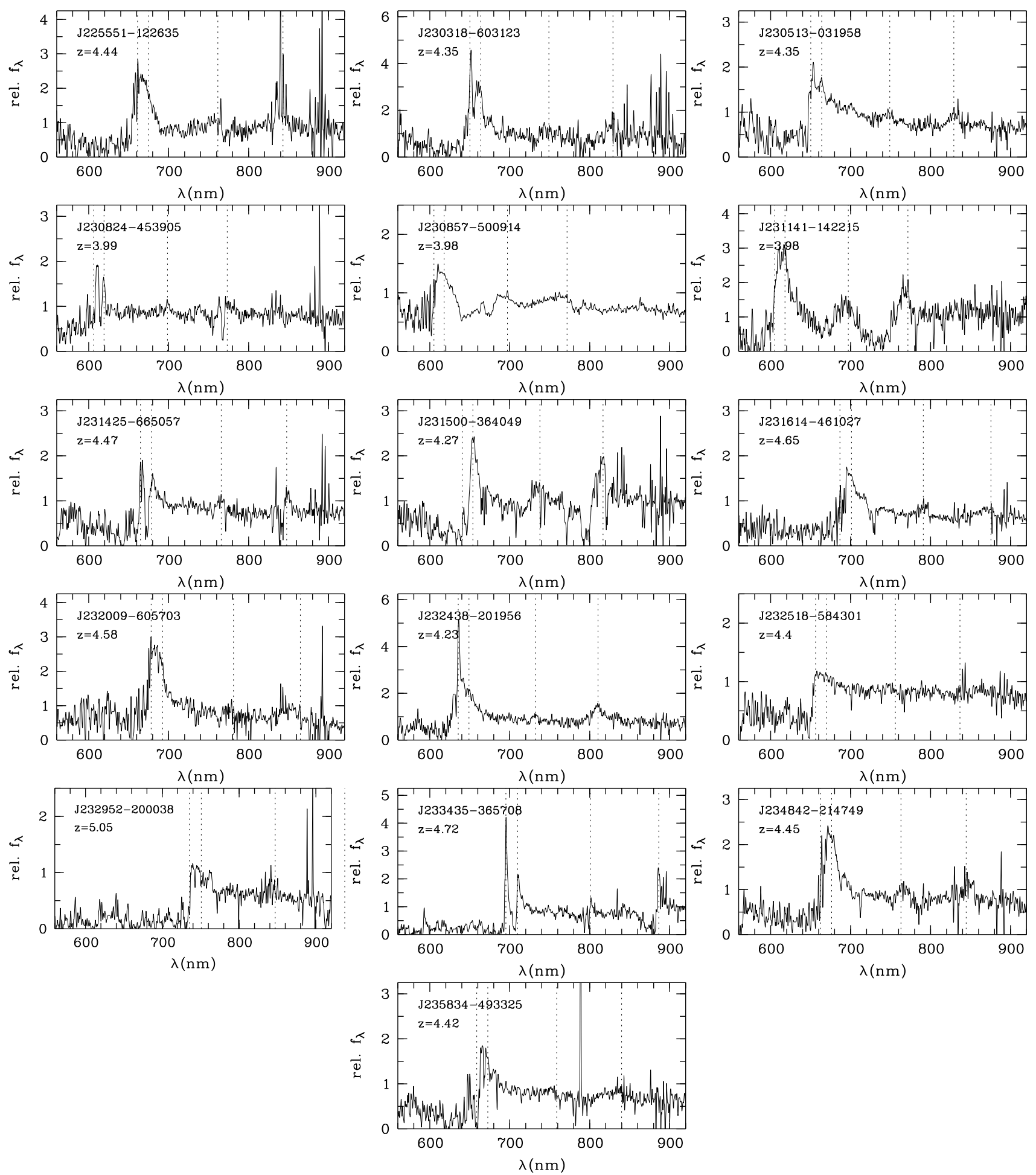

Figure A.7. Gallery of $3.8>z>5.5$ QSO spectra obtained in this work, ordered by RA, page 7 . 


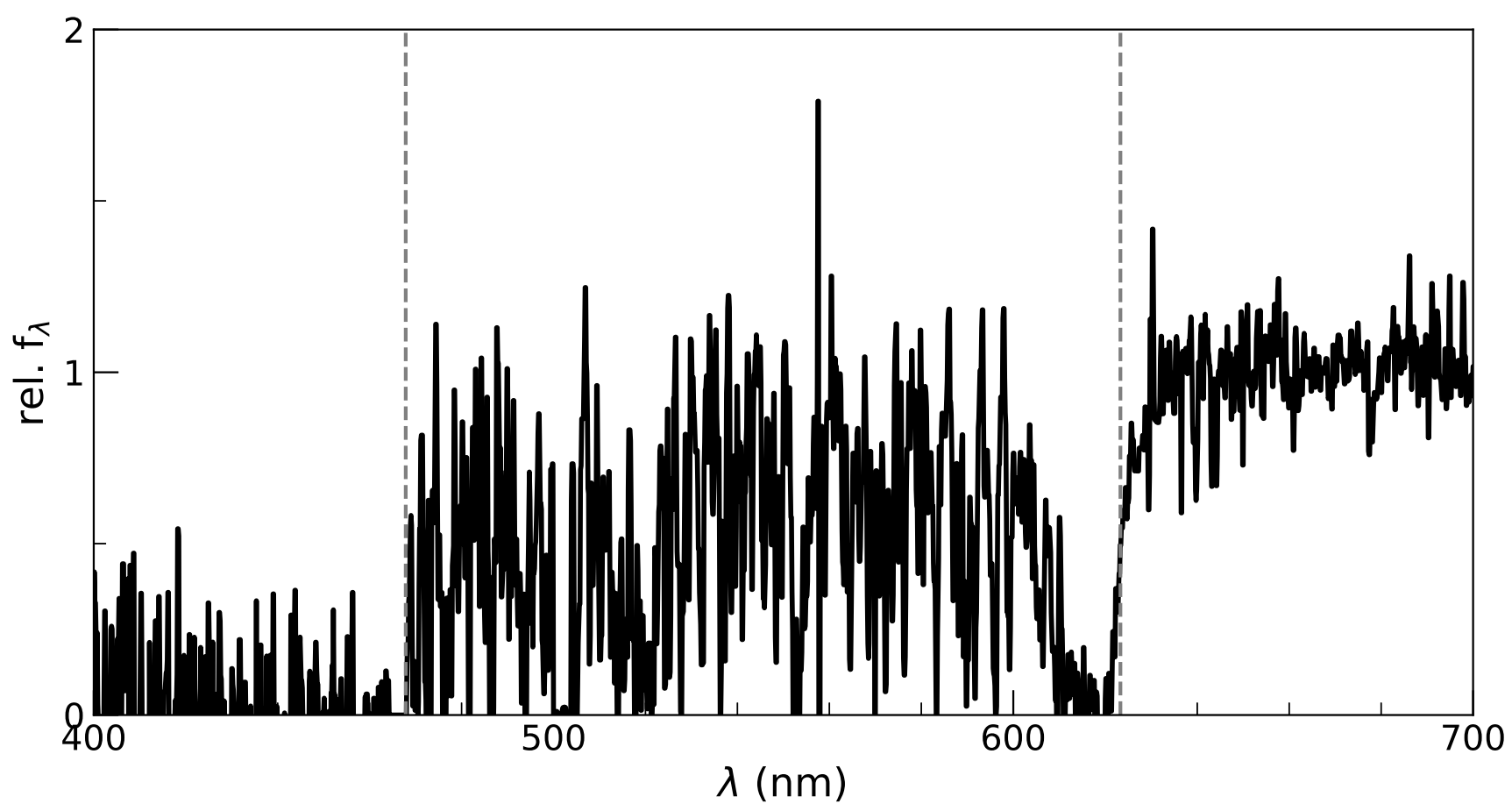

Figure B.1. Coadded observed-frame WiFeS spectrum of SMSS J164147.78-775029.8 obtained with two different beam-splitters to better cover the wavelength range between Ly $\alpha$ and the Lyman limit (shown as the two dashed vertical lines for our adopted redshift of $z=4.13$.).

\section{APPENDIX B: SMSS J164147.78-775029.8}

The lack of prominent emission lines in SMSS J164147.78-775029.8 led us to acquire additional WiFeS spectroscopy with the RT480 beam-splitter on UT 2021-07-04, in an attempt to better examine the associated Ly $\alpha$ forest absorption and confirm the redshift. We present the weighted average spectrum in Figure B.1, with a 3-pixel median smoothing applied to reduce the noise. The onset of absorption shortward of Ly $\alpha$ and the lack of transmitted flux shortward of the Lyman limit lend confidence to the inferred redshift of 4.13.

This paper has been typeset from a $\mathrm{T}_{\mathrm{E}} \mathrm{X} / \mathrm{L} \mathrm{T} \mathrm{E} \mathrm{X}$ file prepared by the author. 